Archive for

Organic Chemistry
Arkivoc 2018, part v, 0-0

to be inserted by editorial office

\title{
Click chemistry-assisted synthesis of novel C-2 triazole-linked betulinic acid conjugates with azidothymidine as potential anti-HIV agents
}

\author{
Anna Yu. Spivak, * Darya A. Nedopekina, Zulfiya R. Galimshina, Rezeda R. Khalitova, \\ Zarema R. Sadretdinova, Rinat R. Gubaidullin, and Victor N. Odinokov \\ Institute of Petrochemistry and Catalysis, Russian Academy of Sciences, \\ 141 prosp. Oktyabrya, 450075 Ufa, Russian Federation \\ Email:spivak.ink@gmail.com
}

Received 05-21-2018

Accepted 07-30-2018

Published on line $08-18-2018$

\section{Abstract}

A new synthetic approach to C-2 triazole-linked bioconjugates of lupane triterpenoids with 3'-azido-3'deoxythymidine (AZT) based on Cu'-catalyzed 1,3-cycloaddition between alkynes and azides is described. The proposed strategy towards AZT-betulinic acid hybrid molecules as potential anti-HIV agents makes it possible to vary the $\mathrm{C}-3$ and $\mathrm{C}-28$ pharmacophores in triterpene moieties. The $\mathrm{C}-2$ propargyl-substituted betulinic acid and its mono- or bi-functional derivatives with side chain at C-3 and/or C-28 positions were successfully synthesized by employing the click reaction.
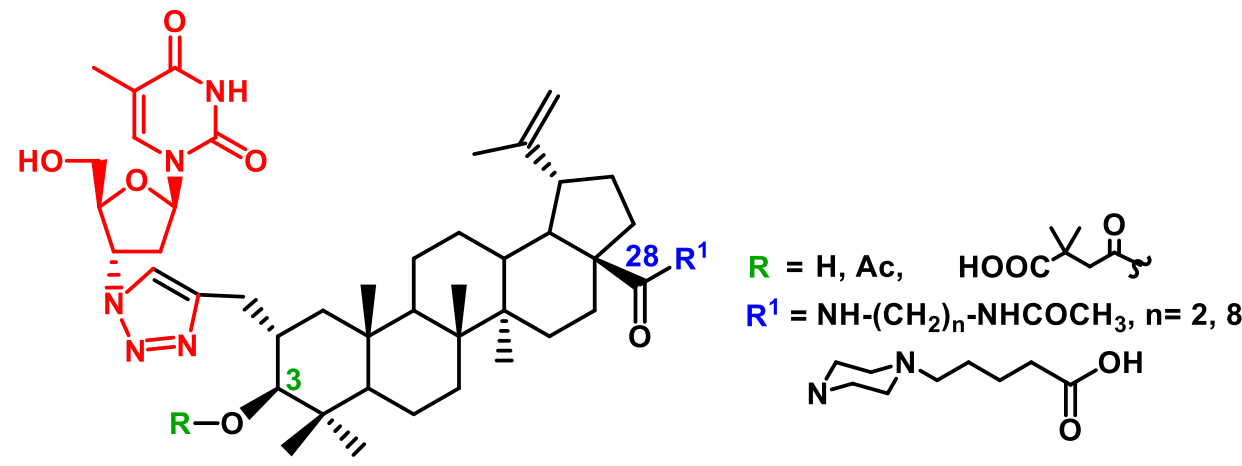

Keywords: Betulinic acid, Bevirimate, AZT, click chemistry, anti-HIV agents. 
The pentacyclic group of lupane triterpenoids (betulin and betulinic acid) represents one of the very important classes of plant natural products that are derived biosynthetically from squalene cyclization. These secondary metabolites possess a wide range of biological effects including antitumor, anti-inflammatory, antibacterial, antimalarial, and antiviral (anti-HIV) activities. ${ }^{1-4}$ The broad spectrum of useful biological properties of lupane triterpenoids is successfully combined with their acceptable systemic toxicity towards animals. Owing to the presence of easily transformable functional groups $(3-\mathrm{OH}, 28-\mathrm{OH}, 28-\mathrm{COOH}, \mathrm{C}-19$ isoprenyl) in the triterpene skeleton, betulin and betulinic acid have a high synthetic potential and are actively used in transformations with the aim to design new drugs. Currently, numerous semisynthetic betulin and betulinic acid derivatives have been prepared; particular attention deserve new potential anti-HIV agents, which show antiviral activity when present in nanomolar concentrations. ${ }^{5-11}$ (Figure 1)

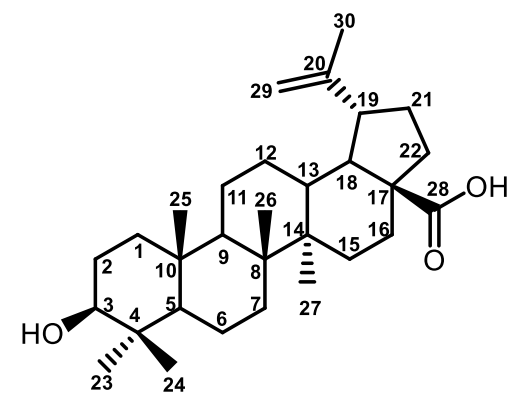

Betulinic acid

$\mathrm{EC}_{50} 1.4 \mu \mathrm{M}$

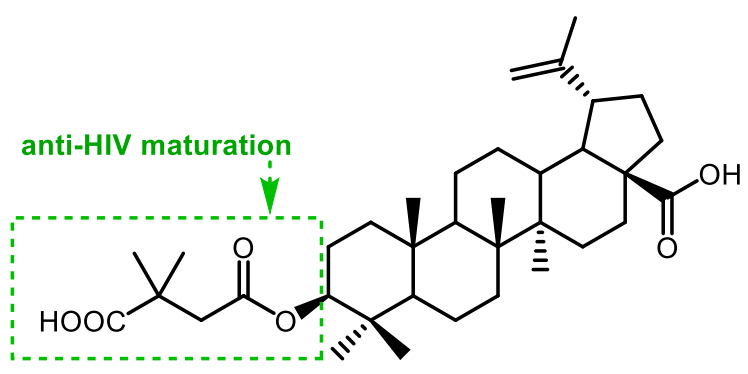

Bevirimat (DSB, PA-457)

$\mathrm{EC}_{50} 9.6 \mathrm{nM}$

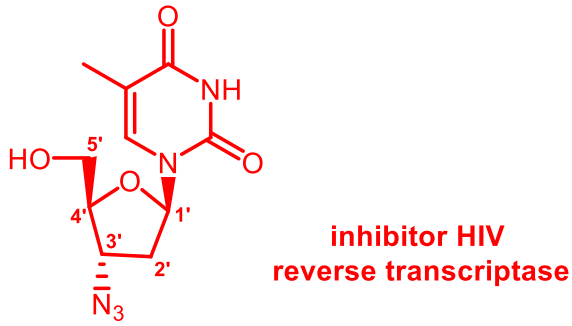

AZT

$\mathrm{EC}_{50} 0.15 \mu \mathrm{M}$

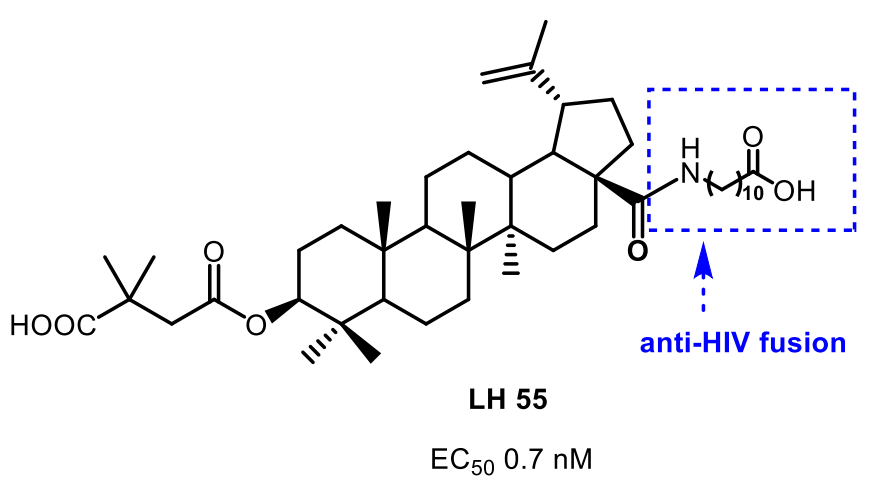

Figure 1. Chemical structures of betulinic acid and derivatives with potent HIV-1 antiviral activity.

The structure-activity relationship studies of anti-HIV-1 lupane triterpenes demonstrated that, in terms of the mechanism of antiviral action, betulinic acid derivatives are subdivided into two types, depending on the structures of C-3 and C-28 pharmacophores. The C-3 acyl derivatives of betulinic or dihydrobetulinic acid are antiretroviral compounds of a new class inhibiting virus protease, which plays a key role in HIV maturation at a late stage of virus replication. 5,6,8 For example, 3-O-(3',3'-dimethylsuccinyl)betulinic acid (DSB, PA-457, MPC4326, or Bevirimat), which is the most promising in this group of anti-HIV agents, prevents cleavage of the capsid protein p25 (CA-sp1) to mature capsid p24 (CA), which results in morphologically defective, noninfectious viral particles. ${ }^{8}$ Unlike Bevirimat and its 3-O-acyl analogues, C-28 amide betulinic acid derivatives act at the initial stage of HIV-1 entry into a human cell, thus preventing the virus fusion with the cell plasmatic membrane. ${ }^{12-14}$ The design of two parallel side chains at the C-3 and C-28 lupane skeleton atoms has resulted 
in bifunctional multi-target antiretroviral agents. This type of lupane triterpenoids showed the best antiviral profile (enhanced viral suppression and control over drug-resistant HIV-1 strains) as compared with the corresponding mono-derivatives of betulin and betulinic acid. ${ }^{15-19}$ The Bevirimat analogues modified at the 28$\mathrm{COOH}$ group acted simultaneously as both HIV-1 entry inhibitors and capsid maturation inhibitors. With the goal to prepare new multi-target antiretroviral drugs, pharmacophore hybridization of betulin and betulinic acid derivatives with AZT (3'-azido-3'-deoxythymidine), the first clinically available nucleoside HIV reverse transcriptase inhibitor, has been performed in several studies. ${ }^{20-23}$ In this case, the combination of two pharmacologically active molecules to hybrid compounds was performed via esterification or copper-catalyzed 1.3-dipolar cycloaddition, with betulin and betulinic acid C-3 and C-28 alkynyl esters or amides acting as the starting reactants. As a result, one or both (C-3 and C-28) pharmacophore groups considerably affecting the antiviral activity were replaced, in the desired products, by AZT residue. We have developed a new synthetic strategy towards triazole-linked AZT bioconjugates with lupane triterpenoids, using betulinic acid derivatives with a terminal acetylenic moiety at the $\mathrm{C}-2$ atom of ring $\mathrm{A}$. The new approach extends the synthetic opportunities for the variation of the $\mathrm{C}-3$ and $\mathrm{C}-28$ side chains in the triterpene moiety of the AZT-triterpenoid hybrids. An efficient method for the synthesis of C-2-propynyl 3-oxo-triterpene acid derivatives has been developed previously by our research team. ${ }^{24}$ The C-2-acetylenic triterpenoid derivatives have been successfully used in the CuACC reactions with sugar azides. ${ }^{24,25}$ This communication reports the synthesis of a group of ten C-2 1,2,3-triazole-linked AZT conjugates with betulinic acid derivatives.

\section{Results and Discussion}

The basic substrate, C-2-propargyl-substituted betulinic acid 4, was prepared from betulinic acid by the method developed by our research team, ${ }^{24}$ with the key steps being $\alpha$-alkylation of potassium enoxytriethylborate, generated from methyl betulonate 2 on treatment with $\mathrm{KN}\left(\mathrm{SiMe}_{3}\right)_{2}-\mathrm{Et}_{3} \mathrm{~B}$ in $\mathrm{DME}_{1}$ with propargyl bromide; stereoselective reduction of the 3-oxo group in triterpenoid 3 with $\mathrm{NaBH}_{4}-\mathrm{CeCl}_{3}$; and the subsequent demethylation of the sterically hindered 28-ester group on treatment with Lil in DMF (Scheme 1).
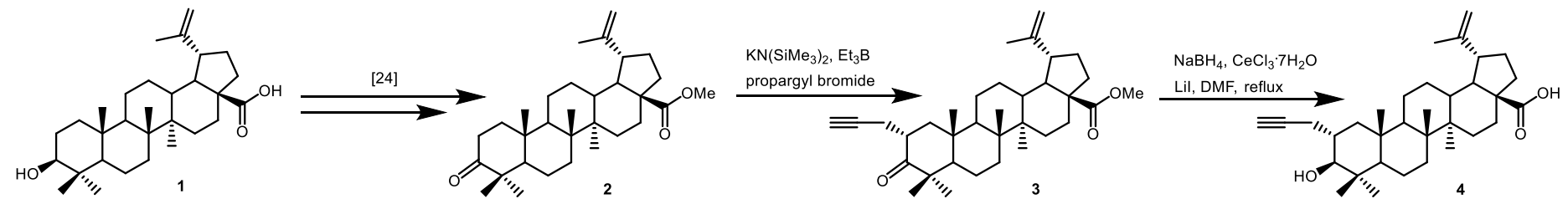

Scheme 1. Synthesis of betulinic acid C-2 propargyl derivatives.

The reaction of triterpenoid 4 with 2,2-dimethylsuccinic anhydride on refluxing in pyridine in the presence of DMAP resulted in the C-2-propynyl analogue of Bevirimat 6 in $40 \%$ yield (Scheme 2).

Acetate 5 obtained upon hydroxyl group protection in triterpenoid 4 was converted to C-28 amide derivatives $\mathbf{8 a - 1 1 a , 1 4}$, and $\mathbf{1 5 a}$ via the unstable acid chloride $\mathbf{7}$. This reaction was carried out for N-Bocprotected or free alkylenediamines with short $(n=2)$ or long $(n=8)$ aminoalkane chains. Amide derivatives $10 a$ and 11a with a primary terminal amino group were converted to $N$-acetyldiaminoalkane derivatives $12 \mathrm{a}$ and 13a by treatment with acetic anhydride in the presence of pyridine and DMAP. It is known from the literature that piperazine moiety is present in many biologically active compounds and pharmaceuticals and is widely used as a promising pharmacophore in the design and synthesis of new potential drugs. ${ }^{26,27}$ The incorporation 
of the piperazine moiety into the C-28 side chain of Bevirimat analogues afforded compounds with high activity against HIV replication affecting Bevirimat-resistant viral strains. ${ }^{12,19}$ We synthesized C-28-piperazine-
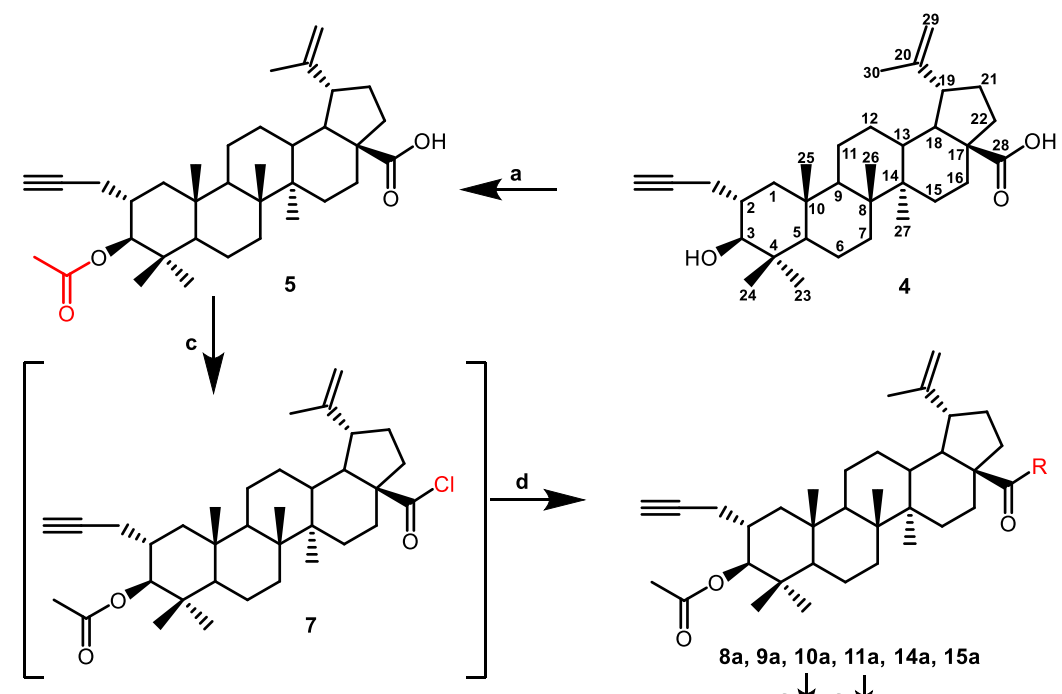

$a \downarrow a \downarrow$

12a 13a

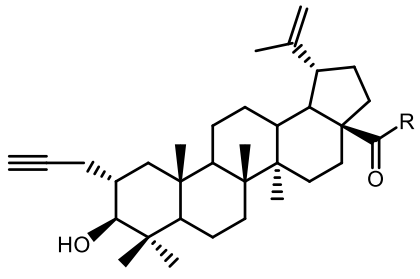

$8 b, 9 b, 12 b, 13 b$

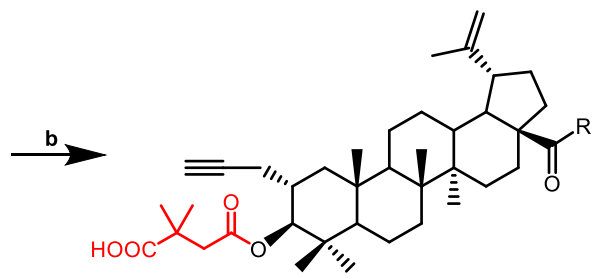

16-19
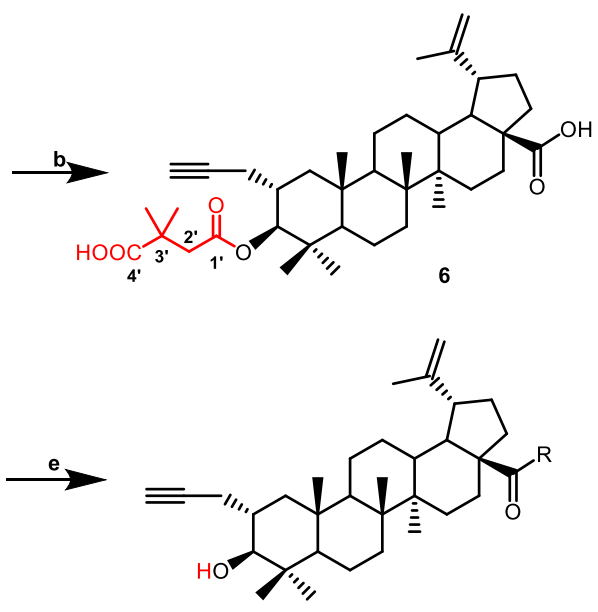

$8 b, 9 b, 12 b, 13 b, 14 b, 15 b$

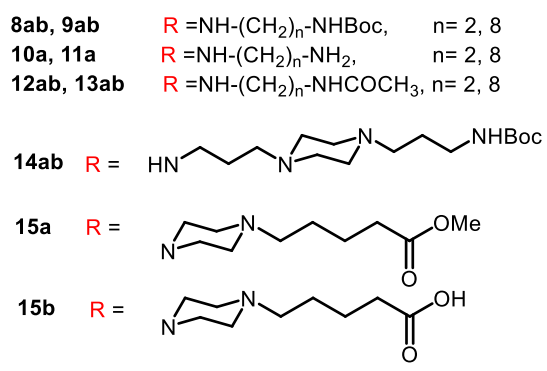

16, $17 \quad \mathrm{R}=\mathrm{NH}-\left(\mathrm{CH}_{2}\right)_{\mathrm{n}}-\mathrm{NHBoc}, \quad \mathrm{n}=2,8$

18, $19 \mathrm{R}=\mathrm{NH}-\left(\mathrm{CH}_{2}\right)_{n}-\mathrm{NHCOCH}_{3}, \mathrm{n}=2,8$

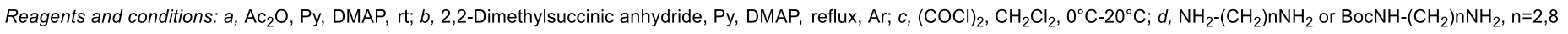
or methyl 5-piperazine pentanoate or N-Boc-bisaminopropylpiperazine, $\mathrm{Et}_{3} \mathrm{~N}, \mathrm{CH}_{2} \mathrm{Cl}_{2}, \mathrm{rt} ; \mathrm{e}, 4 \mathrm{~N} \mathrm{NaOH}, \mathrm{MeOH}, \mathrm{THF}, \mathrm{rt}$

Scheme 2. Synthesis of mono- and bi-functional betulinic acid derivatives with C-2 propargyl substituent.

linked lupane triterpenoids $14 a, 15 a$ by the reaction of chloride 7 with methyl 5-piperazinepentanoate and with N-Boc-protected 1,4-bis-(3-aminopropyl)piperazine. The piperazinepentanoic acid derivative was presynthesized by a reported procedure. ${ }^{12}$ Hydrolysis of the acetate group in compounds $8 \mathbf{a}, 9 \mathbf{a}$, and $12 \mathrm{a}-\mathbf{1 5 a}$ with $4 \mathrm{~N} \mathrm{NaOH}$ in a MeOH-THF mixture furnished triterpenoids $\mathbf{8 b}, \mathbf{9 b}$, and $\mathbf{1 2 b - 1 5 b}$. Some of these compounds (12b, 14b and 15b) were involved in the CuACC reactions with AZT to afford the corresponding hybrid molecules 23-25 (Schemes 2 and 3). Triterpenoids $\mathbf{8 b} \mathbf{6} \mathbf{9 b}, \mathbf{1 2} \mathbf{b}$ and $\mathbf{1 3} \mathbf{b}$ were converted into $\mathrm{C}-3$ dimethylsuccinyl derivatives $\mathbf{1 6 - 1 9}$ in $42 \%-90 \%$ yields under the reaction conditions used for acylation of compound $\mathbf{4}$ into ester $\mathbf{6}$. The reaction of C-2 propargyl betulinic acid derivatives $\mathbf{4}$ and $\mathbf{5}$ with AZT induced by $\mathrm{Cul}$ in $\mathrm{Bu}^{\mathrm{t}} \mathrm{OH}$ at $70{ }^{\circ} \mathrm{C}$ gave target compounds 20 and 21 in $70 \%$ and $55 \%$ yields, after column chromatography on $\mathrm{SiO}_{2}$ (Scheme 3).

However, under these conditions, acetylenic derivatives 6 and 16-19 react with AZT over long periods of time, with the yields of final products being not higher than $32 \%$. The reaction conditions and the yields of conjugates were substantially affected by replacement of the $\mathrm{Bu}^{\mathrm{t} O H}$ solvent by DMSO. With the use of $\mathrm{CuSO}_{4} \cdot 5 \mathrm{H}_{2} \mathrm{O}$ and sodium ascorbate in DMSO, substrates $6, \mathbf{1 2 b}, \mathbf{1 4 b}, \mathbf{1 5 b}$, and 16-19 react with AZT at room 

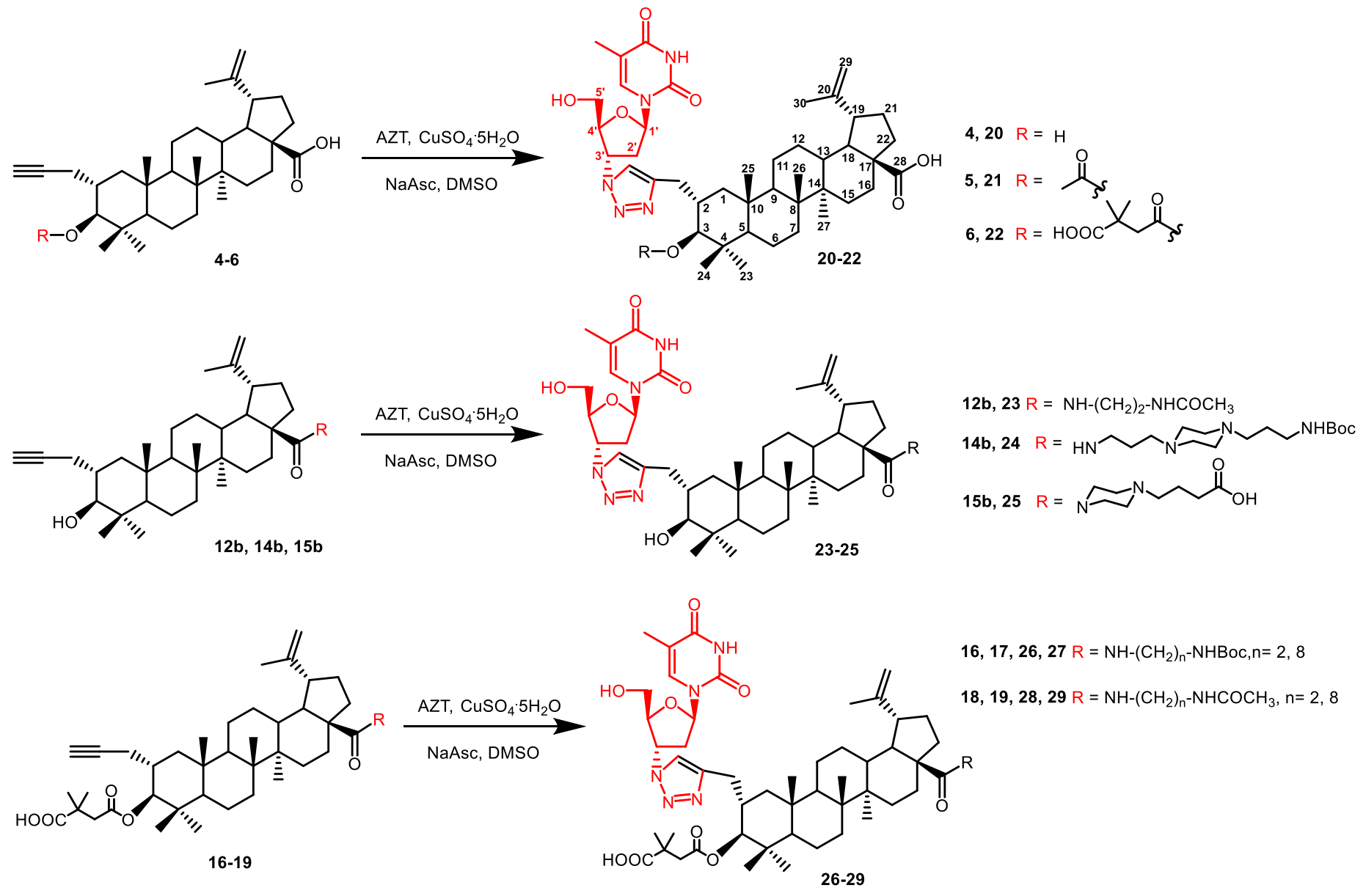

Scheme 3. Synthesis of AZT-triterpenoid conjugates with side chains at C-3 or/and C-28 positions.

are $60 \%-73 \%$ after chromatography on $\mathrm{SiO}_{2}$ for removing the solvent and copper and reactant traces. Our attempts to carry out the CUACC reactions for triterpenoids 10a and 11a with primary amino groups in the side chain were unsuccessful. The bioconjugates were formed in low yields, and isolation by column chromatography on $\mathrm{SiO}_{2}$ was hampered by low chromatographic mobility of the polar compounds. Meanwhile, it follows from previous studies that the use of protected amines (N-Boc amino acids ${ }^{18}$ or $\mathrm{N}$ acetylamines ${ }^{16}$ ) in the design of bifunctional betulinic acid analogues does not have an adverse effect on the antiviral activities of the potential anti-HIV agents in comparison with the prototypes.

The structures of the obtained compounds were confirmed by $1 \mathrm{D}\left({ }^{1} \mathrm{H},{ }^{13} \mathrm{C}, \mathrm{APT}\right)$ and $2 \mathrm{D}$ homo- (COSY, NOESY) and heteronuclear (HSQC, HMBC) experiments. It is known that esterification of betulinic acid with 2,2-dimethylsuccinic anhydride on refluxing in pyridine in the presence of DMAP affords a mixture of 3-0-(3,3'dimethylsuccinyl)- and 3-O-(2,2'-dimethylsuccinyl)betulinic acids, with the 3,3'-dimethylsuccinyl isomer being formed predominantly (95:5 ratio of the regioisomers) $[5,6]$. After purification by chromatography on $\mathrm{SiO}_{2}$, the resulting dimethylsuccinyl derivatives 6, 16-19 and their conjugates 22, 26-29 were individual compounds, as shown by HPLC and NMR spectroscopy. The NMR spectra of compounds 6 and 16-19 showed similar chemical shifts; therefore, we discuss the spectral data for triterpenoid 6 as a typical representative of this series. The structure of the succinyl group of compound 6 was confirmed by the $2 \mathrm{D}\left({ }^{1} \mathrm{H}-{ }^{13} \mathrm{C}\right) \mathrm{HMBC}$ spectra, which showed a correlation of the methylene proton signals at $\delta 2.67$ and $2.73 \mathrm{ppm}$ (two AB type doublets, J $15.0 \mathrm{~Hz}$ ) with the $\mathrm{C}-1^{\prime}$ carbon signal of the carbonyl group at $\delta 171.01 \mathrm{ppm}$. The proton signals for the geminal 
methyl groups Me-3' were correlated with the signal at $183.39 \mathrm{ppm}$, which belonged to the C-4' atom of the carboxyl group. The assignment of the signal with $\delta 171.01 \mathrm{ppm}$ to the $\mathrm{C}-1^{\prime}$ the carbonyl carbon followed from correlation with the $\mathrm{H}-3$ proton signal. The chemical shifts for the atoms of the terpene skeleton were also assigned considering published data. ${ }^{24}$ The formation of triazole rings is evidenced by the characteristic $\underline{\mathrm{CH}}=\mathrm{C}$ $\mathrm{N}$ signals at 7.45-7.90 ppm in the ${ }^{1} \mathrm{H}$ NMR spectra of compounds 20-29. In the ${ }^{13} \mathrm{C}$ NMR spectra, the signals for the $[1,5]$-triazole carbon atoms $\underline{\mathrm{CH}}=\mathrm{C}-\mathrm{N}$ and $\mathrm{CH}=\underline{\mathrm{C}}-\mathrm{N}$ occur at 121.85-123.88 and 145.67-148.10 ppm, respectively.

\section{Conclusions}

Despite the advances made in antiretroviral therapy, there is a vital need for new anti-HIV agents. The challenges of control of the HIV infection are caused by fast appearance of drug-resistant HIV strains and high toxicity of pharmaceutical drugs, which generate numerous adverse effects on long-term administration. Currently, the development of new anti-HIV agents is focused on compounds that could act on two or more molecular targets and be active at different phases of the HIV replication cycle. New opportunities and prospects for the design of multi-target antiviral drugs were offered by the discovery of the $\mathrm{Cu}(\mathrm{I})$-catalyzed azide-alkyne cycloaddition strategy (CuAAC reactions). ${ }^{29}$ The CuAAC reactions or click-reactions are extensively employed in conjugate synthesis and pharmacophore hybridization of various organic compounds, owing to their versatility, high chemoselectivity, and mild conditions. In this study, we have prepared new trisubstituted betulinic acid derivatives containing 3-O-acyl and 28-amide side chains and a propynyl group at the C-2 position of ring $A$ of the lupane core, including compounds $6,15 \mathbf{b}$ and 19 , acetylenic analogues of known triterpenes, $8,12,16$ which are effective against HIV-1. Novel C-2 acetylenic triterpenoids were successfully conjugated with AZT using a click chemistry-based approach.

\section{Experimental Section}

General. IR spectra were obtained with use of a Bruker Vertex 70v spectrometer (thin films or solutions in $\left.\mathrm{CHCl}_{3}\right) \cdot{ }^{1} \mathrm{H}$ and ${ }^{13} \mathrm{C}$ NMR spectra were recorded on a Bruker AVANCE-500 instrument $\left(500.13\left({ }^{1} \mathrm{H}\right)\right.$ and 125.78 $\mathrm{MHz}\left({ }^{13} \mathrm{C}\right)$ ) or on Bruker AVANCE-400 $\left(400.13\left({ }^{1} \mathrm{H}\right)\right.$ and $\left.100.62 \mathrm{MHz}\left({ }^{13} \mathrm{C}\right)\right)$ in $\mathrm{CDCl}_{3}$ or in MeOD with Me $4 \mathrm{Si}$ as the internal standard. Mass spectra of new compounds were recorded on a Bruker-Autoflex III spectrometer (MALDI TOF, positive ion mode, sinapic acid as the matrices). Optical rotation was determined on a PerkinElmer-141 polarimeter. Specific rotation $[\alpha]_{D}$ is expressed in $($ deg $\mathrm{mL}) /(\mathrm{g} \mathrm{dm})$; the concentration of the solution $c$ is expressed in $\mathrm{g} / 100 \mathrm{~mL}$. Elemental analysis was carried out on a Carlo Erba 1106 analyzer. TLC was carried out on Sorbfil plates (Sorbpolimer, Krasnodar, Russia) in hexane-EtOAc and chloroform-methanol, spots were visualized with anisaldehyde. Silica gel L (KSKG grade, 50-160 $\mu \mathrm{m}$ ) was employed for column chromatography. The starting compounds betulin, betulinic acid and reagents: $\mathrm{BEt}_{3}(95 \%), \mathrm{KN}\left(\mathrm{SiMe}_{3}\right)_{2}(1 \mathrm{M}$ solution in THF), propargyl bromide, $\mathrm{Lil}, \mathrm{Cul}, \mathrm{Bu}^{t} \mathrm{OH}, \mathrm{NaBH}_{4}, \mathrm{CeCl}_{3} \cdot 7 \mathrm{H}_{2} \mathrm{O}, \mathrm{Et}_{3} \mathrm{~N}, \mathrm{DMF}, \mathrm{DME}$ (dimethoxyethane), acetic anhydride, oxalylcloride, DMAP, 2,2-dimethylsuccinic anhydride, di-tert-butyl dicarbonate, dimethyl sulfoxide, L-ascorbic acid sodium salt, piperazine, methyl bromovalerate (97\%), 3'-azido-3'-deoxythymidine, ethylenediamine, 1,8-diaminooctane 98\%, 1,4-bis-(3-aminopropyl)piperazine $\geq 99 \%$ (Acros Organics). The intermediates 1-5 were synthesized as we described in earlier paper. ${ }^{24}$ Mono-Boc-protected bis- 
aminopropylpiperazine, Boc-diaminoethane and Boc-diaminooctane and methyl 5-piperazine pentanoate were prepared according to known procedures. ${ }^{12,29}$

General procedure for the preparation of conjugates $8 \mathrm{a}-11 \mathrm{a}, 14 \mathrm{a}$ and $15 \mathrm{a}$. Oxalyl chloride $(0.3 \mathrm{~mL}, 3 \mathrm{mmol})$ was added with stirring to a solution of triterpenoid $5(1 \mathrm{mmol})$ in dry $\mathrm{CH}_{2} \mathrm{Cl}_{2}(5 \mathrm{~mL})$ precooled to $0^{\circ} \mathrm{C}$, and stirring of the reaction mixture was continued at room temperature for $2 \mathrm{~h}$. Then the solvent and excess oxalyl chloride were removed under vacuum. The residue was treated with ethylenediamine $(0.4 \mathrm{~mL}, 6 \mathrm{mmol}), 1,8-$ diaminooctane $(0.87 \mathrm{~g}, 6 \mathrm{mmol}), \mathrm{N}$-Boc-bisaminopropylpiperazine $(0.48 \mathrm{~g}, 1.6 \mathrm{mmol})$, N-Boc-ethylenediamine $(0.26 \mathrm{~g}, 1.6 \mathrm{mmol}), \mathrm{N}$-Boc-1,8-octanediamine $(0.39 \mathrm{~g}, 1.6 \mathrm{mmol})$, or methyl 5-piperazinepentanoate $(0.32 \mathrm{~g}$, $1.6 \mathrm{mmol}$ ) and with triethylamine $(0.2 \mathrm{~mL}, 1.6 \mathrm{mmol})$ in dry $\mathrm{CH}_{2} \mathrm{Cl}_{2}(15 \mathrm{~mL})$, and the mixture was stirred at room temperature for 16-20 $\mathrm{h}$ until no starting material was observed by TLC. The solution was then diluted with $\mathrm{CH}_{2} \mathrm{Cl}_{2}(20 \mathrm{~mL})$ and washed three times with brine and distilled water. The organic layer was dried over anhydrous $\mathrm{MgSO}_{4}$ and concentrated under reduced pressure. The crude products were then chromatographed on silica gel $\left[\mathrm{CHCl}_{3}-\mathrm{MeOH}(100: 1 \rightarrow 5: 1)\right]$ to obtain pure compounds $8 \mathbf{a}-11 \mathrm{a}, 14 \mathrm{a}$ and $15 \mathrm{a}$.

$\boldsymbol{N}$-[3ß-Acetoxy-2 $\alpha$-propargyl-lup-20(29)-en-28-oyl]- $\boldsymbol{N}^{\prime}$-(tert-butoxycarbonyl)-2-ethylamine $\quad(8 \mathrm{a})$. White powder (0.60 g, 89\%), mp 126-128 ${ }^{\circ} \mathrm{C}$. $[\alpha]_{\mathrm{D}}^{19}-24^{\circ}\left(c\right.$ 0.47, $\left.\mathrm{CHCl}_{3}\right)$. IR (solution in $\mathrm{CHCl}_{3}, \mathrm{~cm}^{-1}$ ): $3369,3312(\mathrm{NH})$, $1735,1717,1639$ (C=O). ${ }^{1} \mathrm{H}$ NMR $\left(500 \mathrm{MHz}^{\mathrm{CDCl}}{ }_{3}\right.$ ): $\delta_{\mathrm{H}} 6.35,5.05$ (both br s, 2H, NH), 4.73, 4.59 (both br s, $2 \mathrm{H}$, $\mathrm{H}-29), 4.47$ (d, $1 \mathrm{H}, J 10.0 \mathrm{~Hz}, \mathrm{H}-3), 3.35-3.23\left(\mathrm{~m}, 4 \mathrm{H},-\mathrm{CH}_{2} \mathrm{NH}-,-\mathrm{CH}_{2} \mathrm{NH}-\mathrm{Boc}\right), 3.16-3.10$ (m, 1H, H-19), 2.49$0.80\left(\mathrm{~m}, 26 \mathrm{H}, \mathrm{CH}, \mathrm{CH}_{2}\right.$ in pentacyclic skeleton and propargyl), $2.09\left(\mathrm{~s}, 3 \mathrm{H}, \mathrm{COCH}_{3}\right), 1.68(\mathrm{~s}, 3 \mathrm{H}, \mathrm{H}-30), 1.44(\mathrm{~s}$, $9 \mathrm{H}, \mathrm{C}\left(\mathrm{CH}_{3}\right)_{3}$ in Boc), 0.96, 0.95, 0.89, 0.83, 0.81 (all s, 3H each, $\left.\mathrm{H}-23-\mathrm{H}-27\right) .{ }^{13} \mathrm{C} \mathrm{NMR}\left(125 \mathrm{MHz}, \mathrm{CDCl}_{3}\right): \delta_{\mathrm{c}} 177.2$ (C-28), $171.1\left(\mathrm{COCH}_{3}\right), 156.9$ (CONH-Boc), 150.9 (C-20), 109.4 (C-29), 83.1 (C-3), 82.6 (C in propargyl), 79.6 (C in Boc), 69.6 ( $\mathrm{CH}$ in propargyl), 55.6 (C-17), 55.4 (C-5), 50.6 (C-9), 50.1 (C-18), 46.8 (C-19), 45.0 (C-1), 42.5 (C-14), $40.8\left(\mathrm{CH}_{2}\right.$ in ethylamine, $\left.\mathrm{C}-8\right), 40.4\left(\mathrm{CH}_{2}\right.$ in ethylamine), 38.9 (C-4), 38.4 (C-13), 37.7 (C-22), 37.3 (C-10), 34.3 (C7), 33.6 (C-16), 33.5 (C-2), 30.8 (C-21), 29.4 (C-15), 28.4 ( $\mathrm{CH}_{3}$ in Boc), 28.1 (C-24), 25.6 (C-12), $22.3\left(\mathrm{CH}_{2}\right.$ in propargyl), $21.0\left(\mathrm{COCH}_{3}, \mathrm{C}-11\right), 19.4$ (C-30), 18.3 (C-6), 17.1 (C-26), 16.2 (C-25, C-23), 14.6 (C-27). Anal. Calcd for $\mathrm{C}_{42} \mathrm{H}_{66} \mathrm{~N}_{2} \mathrm{O}_{5}: \mathrm{C}, 74.29 ; \mathrm{H}, 9.80$. Found: $\mathrm{C}, 73.87, \mathrm{H}, 9.76 \%$. MS: $m / z[\mathrm{M}+\mathrm{Na}]^{+}$, found $701.42\left[\mathrm{C}_{42} \mathrm{H}_{66} \mathrm{~N}_{2} \mathrm{O}_{5}\right]^{+}$ requires 701.49 .

$\boldsymbol{N}$-[3及-Acetoxy-2 $\alpha$-propargyl-lup-20(29)-en-28-oyl]- $\boldsymbol{N}^{\prime}$-(tert-butoxycarbonyl)-8-octylamine $\quad(9 a) . \quad W h i t e$ powder $(0.61 \mathrm{~g}, 80 \%), \mathrm{mp} 96-98^{\circ} \mathrm{C} .[\alpha]_{\mathrm{D}}^{22}-23.3^{\circ}\left(\mathrm{c} 0.33, \mathrm{CHCl}_{3}\right)$. IR (solution in $\left.\mathrm{CHCl}_{3}, \mathrm{~cm}^{-1}\right): 3368,3312(\mathrm{NH})$, 1719, 1698, 1638 (C=O). ${ }^{1} \mathrm{H}$ NMR $\left(500 \mathrm{MHz}, \mathrm{CDCl}_{3}\right): \delta_{\mathrm{H}} 5.67-5.65(\mathrm{~m}, 1 \mathrm{H}, \mathrm{NH}) 4.73$ (br s, 1H, H-29), 4.57-5.55 $(\mathrm{m}, 2 \mathrm{H}, \mathrm{H}-29, \mathrm{NH}) 4.46(\mathrm{~d}, 1 \mathrm{H}, \mathrm{J} 10.0 \mathrm{~Hz}, \mathrm{H}-3), 3.29-3.26(\mathrm{~m}, 1 \mathrm{H}, \mathrm{H}-19), 3.16-3.09\left(\mathrm{~m}, 4 \mathrm{H},-\mathrm{CH}_{2} \mathrm{NH}-,-\mathrm{CH}_{2} \mathrm{NH}-\right.$ Boc), $2.50-0.80\left(\mathrm{~m}, 26 \mathrm{H}, \mathrm{CH}, \mathrm{CH}_{2}\right.$ in pentacyclic skeleton, propargyl and $4 \mathrm{H}$ in octylamine) $2.08\left(\mathrm{~s}, 3 \mathrm{H}, \mathrm{COCH}_{3}\right)$, 1.67 (s, 3H, H-30), 1.43 (br s, 9H, $\mathrm{CH}_{3}$ in Boc), 1.33-1.29 (m, 8H, $\mathrm{CH}_{2}$ in octylamine), 0.96, 0.94, 0.88, 0.82, 0.80 (all s, 3H each, H-23-H-27). ${ }^{13} \mathrm{C} \mathrm{NMR} \mathrm{(125} \mathrm{MHz,} \mathrm{CDCl} 3$ ): $\delta_{c} 176.0$ (C-28), 171.1 ( $\left.\mathrm{COCH}_{3}\right), 156.0$ (CONH-Boc), 151.0 (C-20), 109.4 (C-29), 83.1 (C-3), 82.6 (C in propargyl), 79.0 (C in Boc), 69.6 (CH in propargyl), 55.5 (C-17), 55.4 (C-5), 50.6 (C-9), 50.2 (C-18), 46.8 (C-19), 45.0 (C-1), 42.5 (C-14), 40.8 (C-8), 40.6 ( CH $_{2}$ in octylamine), 39.1 (C-4), 38.9 ( $\mathrm{CH}_{2}$ in octylamine), 38.5 (C-13), 37.7 (C-22), 37.3 (C-10), 34.3 (C-7), 33.8 (C-2), 33.5 (C-16), 30.8 (C21), 30.0 ( $\mathrm{CH}_{2}$ in octylamine), 29.8 (C-15), $29.4\left(\mathrm{CH}_{2}\right.$ in octylamine), $29.2\left(\mathrm{CH}_{2}\right.$ in octylamine), $28.4\left(\mathrm{CH}_{3}\right.$ in $\left.\mathrm{Boc}\right)$, 28.1 (C-24), 26.9, 26.7 ( $\mathrm{CH}_{2}$ in octylamine), 25.6 (C-12), $22.3\left(\mathrm{CH}_{2}\right.$ in propargyl), $21.0\left(\mathrm{COCH}_{3}, \mathrm{C}-11\right), 19.4$ (C-30), 18.3 (C-6), 17.1 (C-26), 17.0 (C-23), 16.2 (C-25), 14.6 (C-27). Anal. Calcd for $\mathrm{C}_{48} \mathrm{H}_{78} \mathrm{~N}_{2} \mathrm{O}_{5}: \mathrm{C}, 75.54 ; \mathrm{H}, 10.30$. Found: $\mathrm{C}, 75.48, \mathrm{H}, 9.97 \%$. MS: $m / z[\mathrm{M}+\mathrm{Na}]^{+}$, found $785.36\left[\mathrm{C}_{48} \mathrm{H}_{78} \mathrm{~N}_{2} \mathrm{O}_{5}\right]^{+}$requires 785.58 .

2-Aminoethyl-3ß-acetoxy-2 $\alpha$-propargyl-lup-20(29)-en-28-oate (10a). White powder (0.43 g, 74\%), mp 152$154{ }^{\circ} \mathrm{C} .[\alpha]_{\mathrm{D}}^{18}-23^{\circ}\left(\mathrm{c} 0.53, \mathrm{CHCl}_{3}\right)$. IR (solution in $\left.\mathrm{CHCl}_{3}, \mathrm{~cm}^{-1}\right)$ : $3309(\mathrm{NH}), 1733,1638(\mathrm{C}=0) .{ }^{1} \mathrm{H} \mathrm{NMR}(500 \mathrm{MHz}$, $\mathrm{CDCl}_{3}$ ): $\delta_{\mathrm{H}} 6.17(\mathrm{t}, 1 \mathrm{H}, J 5 \mathrm{~Hz}, \mathrm{NH}$ ), 4.73, 4.58 (both br s, 2H, H-29), 4.46 (d, 1H, J $10.0 \mathrm{~Hz}, \mathrm{H}-3$ ), 3.37-3.31, 3.273.22 (1H each, $\mathrm{m},-\mathrm{CH}_{2} \mathrm{NH}-$ ), 3.15-3.10 (m, $\left.1 \mathrm{H}, \mathrm{H}-19\right), 2.82\left(\mathrm{t}, 2 \mathrm{H}, J 10.0 \mathrm{~Hz},-\mathrm{CH}_{2} \mathrm{NH}_{2}\right), 2.49-0.80(\mathrm{~m}, 26 \mathrm{H}, \mathrm{CH}$, 
$\mathrm{CH}_{2}$ in pentacyclic skeleton and propargyl), $2.08\left(\mathrm{~s}, 3 \mathrm{H}, \mathrm{COCH}_{3}\right), 1.67(\mathrm{~s}, 3 \mathrm{H}, \mathrm{H}-30), 0.96,0.94,0.88,0.81,0.80$

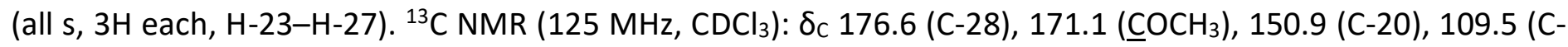
29), 83.1 (C-3), 82.6 (C in propargyl), 69.7 ( $\mathrm{CH}$ in propargyl), 55.7 (C-17), 55.4 (C-5), 50.6 (C-9), 50.1 (C-18), 46.8 (C-19), 45.0 (C-1), 42.5 (C-14), 41.7, $41.6\left(\mathrm{CH}_{2}\right.$ in ethylamine), 40.8 (C-4), 38.9 (C-8), 38.5 (C-13), 37.7 (C-22), 37.3 (C-10), 34.3 (C-7), 33.7 (C-2), 33.5 (C-16), 30.8 (C-21), 29.4 (C-15), 28.1 (C-24), 25.6 (C-12), 22.3 (CH propargyl), $21.03\left(\mathrm{COCH}_{3}, \mathrm{C}-11\right), 19.4$ (C-30), 18.3 (C-6), 17.1 (C-26), 16.3 (C-23), 16.2 (C-25), 14.6 (C-27). Anal. Calcd for $\mathrm{C}_{37} \mathrm{H}_{58} \mathrm{~N}_{2} \mathrm{O}_{3}:$ C, 76.77; $\mathrm{H}$, 10.10. Found: C, 76.87, H, 9.96\%. MS: $\mathrm{m} / z$ [M+Na] $]^{+}$found 601.73 $\left[\mathrm{C}_{37} \mathrm{H}_{58} \mathrm{~N}_{2} \mathrm{O}_{3}\right]^{+}$requires 601.43 .

8-Aminooctyl-3 $\beta$-acetoxy-2 $\alpha$-propargyl-lup-20(29)-en-28-oate (11a). White powder (0.53 g, 80\%), mp 108$110{ }^{\circ} \mathrm{C} .[\alpha]_{\mathrm{D}}^{22}-21^{\circ}\left(c 0.53, \mathrm{CHCl}_{3}\right.$ ). IR (solution in $\left.\mathrm{CHCl}_{3}, \mathrm{~cm}^{-1}\right): 3311(\mathrm{NH}), 1733,1636(\mathrm{C}=\mathrm{O}) .{ }^{1} \mathrm{H} \mathrm{NMR}(500 \mathrm{MHz}$, $\mathrm{CDCl}_{3}$ ): $\delta_{\mathrm{H}} 5.63(\mathrm{t}, 1 \mathrm{H}, J 5 \mathrm{~Hz}, \mathrm{NH}$ ), 4.73, 4.58 (both br s, 2H, H-29), 4.47 (d, 1H, J $10.0 \mathrm{~Hz}, \mathrm{H}-3$ ), 3.31-3.26 (m, $1 \mathrm{H}$, $-\mathrm{CH}_{2} \mathrm{NH}-$ ), 3.18-3.13 (m, 2H, $\left.-\mathrm{CH}_{2} \mathrm{NH}-, \mathrm{H}-19\right), 2.68$ (t, $\left.2 \mathrm{H}, \mathrm{J} 10.0 \mathrm{~Hz},-\mathrm{CH}_{2} \mathrm{NH}_{2}\right), 2.50-0.80\left(\mathrm{~m}, 26 \mathrm{H}, \mathrm{CH}, \mathrm{CH}_{2}\right.$ in pentacyclic skeleton and propargyl), $2.09\left(\mathrm{~s}, 3 \mathrm{H}, \mathrm{COCH}_{3}\right), 1.67(\mathrm{~s}, 3 \mathrm{H}, \mathrm{H}-30), 1.32-1.26\left(\mathrm{~m}, 12 \mathrm{H}, \mathrm{CH}_{2} \mathrm{in}\right.$ octylamine), $0.95,0.92,0.86,0.81,0.78$ (all s, 3H each, $\mathrm{H}-23-\mathrm{H}-27) .{ }^{13} \mathrm{C} \mathrm{NMR}\left(125 \mathrm{MHz}, \mathrm{CDCl}_{3}\right): \delta_{\mathrm{C}} 175.9$ (C-28), $171.1\left(\mathrm{COCH}_{3}\right), 151.0$ (C-20), 109.4 (C-29), 83.1 (C-3), 82.6 (C in propargyl), 69.6 (CH in propargyl), 55.5 (C-17), 55.4 (C-5), 50.6 (C-9), 50.2 (C-18), 46.8 (C-19), 45.0 (C-1), 42.5 (C-14), 42.2 ( $\mathrm{CH}_{2}$ in octylamine), 40.8 (C-8), 39.2 (C-4), 38.9 ( $\mathrm{CH}_{2}$ in octylamine), 38.5 (C-13), 33.8 ( $\mathrm{CH}_{2}$ in octylamine), 37.7 (C-22), 37.3 (C-10), 34.3 (C-7), 33.9 (C-2), 33.5 (C-16), 30.8 (C-21), 29.9 (C-15), 29.4, $29.3\left(\mathrm{CH}_{2}\right.$ in octylamine), 28.1 (C-24), 27.0, $26.8\left(\mathrm{CH}_{2}\right.$ in octylamine), 25.6 (C-12), 22.3 ( $\mathrm{CH}_{2}$ in propargyl), 21.0 ( $\left.\mathrm{COCH}_{3}, \mathrm{C}-11\right), 19.4$ (C-30), 18.4 (C-6), 17.1 (C-26), 17.0 (C-23), 16.2 (C-25), 14.6 (C-27). Anal. Calcd for $\mathrm{C}_{43} \mathrm{H}_{70} \mathrm{~N}_{2} \mathrm{O}_{3}$ : C, 77.89; $\mathrm{H}, 10.64$. Found: $\mathrm{C}, 78.04, \mathrm{H}, 10.57 \%$. MS: $\mathrm{m} / \mathrm{z}[\mathrm{M}+\mathrm{H}]^{+}$, found $663.28\left[\mathrm{C}_{43} \mathrm{H}_{70} \mathrm{~N}_{2} \mathrm{O}_{3}\right]^{+}$requires 663.55 .

$\boldsymbol{N}$-[3ß-Acetoxy-2 $\alpha$-propargyl-lup-20(29)-en-28-oyl]- $\boldsymbol{N}^{\prime}$-(tert-butoxycarbonyl)-\{3-[4-(3-aminopropyl)piperazinyl]\}propylamine (14a). White powder $(0.68 \mathrm{~g}, 83 \%) . \mathrm{mp} 110-112^{\circ} \mathrm{C}$. $[\alpha]_{\mathrm{D}}^{22}-21^{\circ}\left(\mathrm{c} 0.50, \mathrm{CHCl}_{3}\right)$. IR (solution in $\mathrm{CHCl}_{3}, \mathrm{~cm}^{-1}$ ): 3367, $3310(\mathrm{NH}), 1733,1699,1639$ (C=O). ${ }^{1} \mathrm{H} \mathrm{NMR}\left(500 \mathrm{MHz}, \mathrm{CDCl}_{3}\right): \delta_{\mathrm{H}} 6.94(\mathrm{t}, 1 \mathrm{H}, J 5 \mathrm{~Hz}, \mathrm{NH})$, 5.42 (br s, $1 \mathrm{H}, \mathrm{NH}$ ), 4.70, 4.55 (both br s, 2H, H-29), 4.44 (d, 1H, J $10.0 \mathrm{~Hz}, \mathrm{H}-3$ ), 3.32-3.26, 3.15-3.14 (both m, $\left.5 \mathrm{H},-\mathrm{CH}_{2} \mathrm{NH}-,-\mathrm{CH}_{2} \mathrm{NH}-\mathrm{Boc}, \mathrm{H}-19\right), 2.51-2.38\left(\mathrm{~m}, 12 \mathrm{H}, \mathrm{CH}_{2}\right.$ in piperazine and propylamine), 2.19-0.78 (m, 26H, $\mathrm{CH}, \mathrm{CH}_{2}$ in pentacyclic skeleton, propargyl and $4 \mathrm{H}$ in propylamine), $2.06\left(\mathrm{~s}, 3 \mathrm{H}, \mathrm{COCH}_{3}\right), 1.65(\mathrm{~s}, 3 \mathrm{H}, \mathrm{H}-30), 1.41$ (br s, 9H, $\mathrm{CH}_{3}$ in Boc), 0.94, 0.92, 0.86, 0.78, 0.75 (all s, 3H each, H-23-H-27). ${ }^{13} \mathrm{C} \mathrm{NMR}\left(125 \mathrm{MHz}^{\mathrm{C}} \mathrm{CDCl}_{3}\right): \delta_{\mathrm{c}}$ 176.2 (C-28), 171.1 ( $\left.\mathrm{COCH}_{3}\right), 156.0$ (CONH-Boc), 151.0 (C-20), 109.3 (C-29), 83.1 (C-3), 82.5 (C in propargyl), 78.5 (C in Boc), 69.7 ( $\mathrm{CH}$ in propargyl), 58.0, 56.8 ( $\mathrm{CH}_{2}$ in propylamine), 55.4 (C-17), 55.3 (C-5), 53.5, 53.1 ( $\mathrm{CH}_{2}$ in piperazine), 50.6 (C-9), 50.2 (C-18), 46.7 (C-19), 44.9 (C-1), 42.4 (C-14), 40.8 (C-8), 39.9 ( $\mathrm{CH}_{2}$ in propylamine), 39.5 (C-4), 38.9 ( $\mathrm{CH}_{2}$ in propylamine), 38.5 (C-13), 37.5 (C-22), 37.2 (C-10), 34.3 (C-7), 33.8 (C-2), 33.5 (C-16), 30.8 (C-21), 29.4 (C-15), 28.4 ( $\underline{\mathrm{CH}}_{3}$ in Boc), 28.1 (C-24), $26.4\left(\mathrm{CH}_{2}\right.$ in propylamine), $25.5(\mathrm{C}-12), 25.1\left(\mathrm{CH}_{2}\right.$ in propylamine), 22.3 ( $\mathrm{CH}_{2}$ in propargyl), $21.0\left(\mathrm{COCH}_{3}, \mathrm{C}-11\right), 19.4$ (C-30), 18.3 (C-6), 17.1 (C-26), 17.0 (C-23), 16.2 (C-25), 14.5 (C-27). Anal. Calcd for $\mathrm{C}_{50} \mathrm{H}_{82} \mathrm{~N}_{4} \mathrm{O}_{5}: \mathrm{C}, 73.31 ; \mathrm{H}, 10.09$. Found: $\mathrm{C}, 73.24, \mathrm{H}, 9.87 \% . \mathrm{MS}: \mathrm{m} / z$ [M+H] , found $819.52\left[\mathrm{C}_{50} \mathrm{H}_{82} \mathrm{~N}_{4} \mathrm{O}_{5}\right]^{+}$requires 819.64.

Methyl $\mathbf{N}$-[3ß-acetoxy-2 $\alpha$-propargyl-lup-20(29)-en-28-oyl]-5-piperazinylpentanoate (15a). White powder $(0.56 \mathrm{~g}, 78 \%), \mathrm{mp} 116-118{ }^{\circ} \mathrm{C} .[\alpha]_{\mathrm{D}}^{18}-25.45^{\circ}$ (c 0.64, $\left.\mathrm{CHCl}_{3}\right)$. IR (solution in $\mathrm{CHCl}_{3}, \mathrm{~cm}^{-1}$ ): 1736, $1632(\mathrm{C}=0) .{ }^{1} \mathrm{H}$ NMR $\left(500 \mathrm{MHz}, \mathrm{CDCl}_{3}\right.$ ): $\delta_{\mathrm{H}} 4.70,4.54$ (both br s, 2H, H-29), 4.45 (d, 1H, J $\left.10.0 \mathrm{~Hz}, \mathrm{H}-3\right), 3.65\left(\mathrm{~s}, 3 \mathrm{H}, \mathrm{COOCH}_{3}\right.$ ), 2.99-2.94 (m, 1H, H-19), 2.89-2.85 (m, 1H, H-13), 2.44-2.30 (m, 8H, piperazine) 2.19-0.78 (m, 25H, CH, $\mathrm{CH}_{2}$ in pentacyclic skeleton, propargyl, $8 \mathrm{H}$ in pentanoate), $2.06\left(\mathrm{~s}, 3 \mathrm{H}, \mathrm{COCH}_{3}\right), 1.65(\mathrm{~s}, 3 \mathrm{H}, \mathrm{H}-30), 0.93,0.92,0.87$,

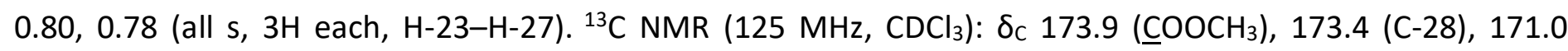
$\left(\mathrm{COCH}_{3}\right), 151.3$ (C-20), 109.2 (C-29), 86.8 (C in propargyl), 83.1 (C-3), 73.6 (CH in propargyl), 57.9, 55.4 ( $\mathrm{CH}_{2} \mathrm{in}$ pentanoate), 54.5 (C-17), $53.4\left(\mathrm{CH}_{2}\right.$ in piperazine), 52.6 (C-18), 51.5 ( $\left.\mathrm{COOCH}_{3}\right), 50.8$ (C-9), 45.7 (C-19), 45.0 (C- 
1), 41.9 (C-14), 40.7 (C-4), 38.9 (C-8), 38.4 (C-2), 37.3 (C-10), 36.8 (C-13), 35.9 (C-22), 34.3 (C-7), 33.8 (CH piperazine), 32.5 (C-16), 31.2 (C-21), 29.7 (C-15), 28.1 (C-24), 26.1 ( $\mathrm{CH}_{2}$ in pentanoate), 25.3 (C-12), $22.3\left(\mathrm{CH}_{2}\right.$ in propargyl), 22.8 ( $\mathrm{CH}_{2}$ in pentanoate), 21.2 (C-11), $21.0(\mathrm{COCH} 3), 19.6$ (C-30), 18.3 (C-6), 17.1 (C-26), 17.0 (C-23), 16.1 (C-25), 14.6 (C-27). Anal. Calcd for $\mathrm{C}_{45} \mathrm{H}_{70} \mathrm{~N}_{2} \mathrm{O}_{5}: \mathrm{C}, 75.17 ; \mathrm{H}, 9.81$. Found: C, 75.21, H, 9.74\%. MS: $\mathrm{m} / z$ [M$\mathrm{H}]^{+}$, found $717.36\left[\mathrm{C}_{45} \mathrm{H}_{70} \mathrm{~N}_{2} \mathrm{O}_{5}\right]^{+}$requires 717.52 .

General procedure for the preparation of acetamides $12 \mathrm{a}$ and $13 \mathrm{a}$. To a solution of the above amine intermediate 10 a or $11 \mathrm{a}(1 \mathrm{mmol})$ in anhydrous pyridine $(4 \mathrm{~mL})$ were added acetic anhydride $(0.2 \mathrm{~mL}, 2 \mathrm{mmol})$, DMAP $(0.12 \mathrm{~g}, 1 \mathrm{mmol})$ and stirring of the reaction mixture was continued at room temperature for $2 \mathrm{~h}$ (monitoring by TLC). Then the mixture was diluted with $\mathrm{H}_{2} \mathrm{O}(10 \mathrm{~mL})$ and extracted with EtOAc $(4 \times 10 \mathrm{~mL})$. The combined extracts were washed with brine, dried over anhydrous $\mathrm{MgSO}_{4}$, and concentrated under reduced pressure. The crude products were then chromatographed on silica gel $\left[\mathrm{CHCl}_{3}-\mathrm{MeOH}(100: 1)\right]$ to obtain pure compounds 10a and 13a.

\{[N-(3ß-Acetoxy-2 $\alpha$-propargyl-lup-20(29)-en-28-oyl)-2-aminoethyl]carbamoyl\}methane (12a). White powder (0.56 g, 90\%), mp 148-150 ${ }^{\circ} \mathrm{C} .[\alpha]_{\mathrm{D}}^{18}-24^{\circ}\left(\mathrm{c} 0.44, \mathrm{CHCl}_{3}\right.$ ). IR (solution in $\mathrm{CHCl}_{3}, \mathrm{~cm}^{-1}$ ): $3310(\mathrm{NH}), 1734,1718$, $1637(\mathrm{C}=0) .{ }^{1} \mathrm{H}$ NMR $\left(500 \mathrm{MHz}, \mathrm{CDCl}_{3}\right.$ ): $\delta_{\mathrm{H}} 6.74,6.49$ (both br s, 2H, NH), 4.72, 4.59 (both br s, 2H, H-29), 4.46 (d, $1 \mathrm{H}, \mathrm{J} 10.0 \mathrm{~Hz}, \mathrm{H}-3), 3.39-3.32\left(\mathrm{~m}, 4 \mathrm{H},-\mathrm{CH}_{2} \mathrm{NH}-, \mathrm{CH}_{2} \mathrm{NHCOCH}_{3}\right), 3.12-3.07(\mathrm{~m}, 1 \mathrm{H}, \mathrm{H}-19), 2.47-0.80(\mathrm{~m}, 26 \mathrm{H}$, $\mathrm{CH}, \mathrm{CH}_{2}$ in pentacyclic skeleton and propargyl), $2.09\left(\mathrm{~s}, 3 \mathrm{H}, \mathrm{COCH}_{3}\right), 1.99\left(\mathrm{~s}, 3 \mathrm{H},-\mathrm{NHCOCH}_{3}\right), 1.68(\mathrm{~s}, 3 \mathrm{H}, \mathrm{H}-30)$, 0.96, 0.93, 0.88, 0.82, 0.80 (all s, 3H each, $\mathrm{H}-23-\mathrm{H}-27) .{ }^{13} \mathrm{C} \mathrm{NMR} \mathrm{(125} \mathrm{MHz,} \mathrm{CDCl} 3$ ): $\delta_{\mathrm{c}} 178.1$ (C-28), 171.3 $\left(\mathrm{NH}_{\mathrm{COCH}}\right), 171.1\left(\mathrm{COCH}_{3}\right), 150.7$ (C-20), 109.6 (C-29), 83.0 (C-3), 82.5 (C in propargyl), 69.7 (CH in propargyl), 55.6 (C-17), 55.3 (C-5), 50.5 (C-9), 50.0 (C-18), 46.8 (C-19), 44.9 (C-1), 42.5 (C-14), 40.8 ( $\mathrm{CH}_{2}$ in ethylamine, C-4), $39.7\left(\mathrm{CH}_{2}\right.$ in ethylamine), 38.9 (C-8), 38.4 (C-13), 37.7 (C-22), 37.2 (C-10), 34.2 (C-7), 33.5 (C-2), 33.5 (C-16), 30.8 (C-21), 29.4 (C-15), 28.1 (C-24), 25.5 (C-12), $23.2\left(\mathrm{NHCOCH}_{3}\right), 22.3\left(\mathrm{CH}_{2}\right.$ in propargyl), $21.0\left(\mathrm{COCH} 3 \underline{\mathrm{C}}_{3}, \mathrm{C}-11\right)$, 19.3 (C-30), 18.3 (C-6), 17.1 (C-26), 17.0 (C-23), 16.1 (C-25), 14.6 (C-27). Anal. Calcd for $\mathrm{C}_{39} \mathrm{H}_{60} \mathrm{~N}_{2} \mathrm{O}_{4}: \mathrm{C}, 75.44 ; \mathrm{H}$, 9.74. Found: $\mathrm{C}, 74.38, \mathrm{H}, 9.69 \%$. MS: $m / z[\mathrm{M}+\mathrm{Na}]^{+}$, found $643.37\left[\mathrm{C}_{39} \mathrm{H}_{60} \mathrm{~N}_{2} \mathrm{O}_{4}\right]^{+}$requires 643.45 .

$\{[N$-(3ß-Acetoxy-2 $\alpha$-propargyl-lup-20(29)-en-28-oyl)-8-aminooctyl]carbamoyl\}methane (13a). White powder (0.56 g, 80\%), mp 106-108 ${ }^{\circ} \mathrm{C} .[\alpha]_{\mathrm{D}}^{22}-23^{\circ}\left(\mathrm{c} 0.48, \mathrm{CHCl}_{3}\right)$. IR (solution in $\left.\mathrm{CHCl}_{3}, \mathrm{~cm}^{-1}\right): 3311(\mathrm{NH}), 1733(\mathrm{C}=0) .{ }^{1} \mathrm{H}$ NMR $\left(500 \mathrm{MHz}, \mathrm{CDCl}_{3}\right): \delta_{\mathrm{H}}$ 5.77-5.71 (m, 2H, NH), 4.72, 4.57 (both br s, 2H, H-29), $4.46(\mathrm{~d}, 1 \mathrm{H}, J 10.0 \mathrm{~Hz}, \mathrm{H}-3$ ), 3.22-3.14 (m, 5H, $\left.-\mathrm{CH}_{2} \mathrm{NH}-, \mathrm{CH}_{2} \mathrm{NHCOCH}_{3}, \mathrm{H}-19\right), 2.47-0.80\left(\mathrm{~m}, 26 \mathrm{H}, \mathrm{CH}, \mathrm{CH}_{2}\right.$ in pentacyclic skeleton and propargyl), $2.08\left(\mathrm{~s}, 3 \mathrm{H}, \mathrm{COCH}_{3}\right), 1.97\left(\mathrm{~s}, 3 \mathrm{H},-\mathrm{NHCOCH}_{3}\right), 1.67(\mathrm{~s}, 3 \mathrm{H}, \mathrm{H}-30), 1.37-1.30\left(\mathrm{~m}, 12 \mathrm{H}, \mathrm{CH}_{2}\right.$ in octylamine), 0.96, 0.94, 0.88, 0.82, 0.80 (all s, 3H each, $\mathrm{H}-23-\mathrm{H}-27) .{ }^{13} \mathrm{C} \mathrm{NMR}\left(125 \mathrm{MHz}, \mathrm{CDCl}_{3}\right): \delta_{\mathrm{c}} 176.0$ (C-28), $171.1\left(\mathrm{COCH}_{3}\right), 170.0\left(\mathrm{NHC}_{\mathrm{COCH}}\right), 151.0$ (C-20), 109.4 (C-29), 83.1 (C-3), 82.6 (C in propargyl), 69.7 (CH in propargyl), 55.5 (C-17), 55.4 (C-5), 50.6 (C-9), 50.1 (C-18), 46.8 (C-19), 45.0 (C-1), 42.5 (C-14), 40.8 (C-8), 39.6 ( $\mathrm{CH}_{2}$ in octylamine), 39.1 (C-4), 38.9 ( $\mathrm{CH}_{2}$ in octylamine), 38.5 (C-13), 37.7 (C-22), 37.3 (C-10), 34.3 (C-7), 33.8 (C-2), 33.5 (C-16), 30.8 (C-21), 29.8 ( $\mathrm{CH}_{2}$ in octylamine), 29.6 (C-15), $29.4\left(\mathrm{CH}_{2}\right.$ in octylamine), $29.1\left(\mathrm{CH}_{2}\right.$ in octylamine), 28.1 (C-24), $26.8\left(\mathrm{CH}_{2}\right.$ in octylamine), 25.6 (C-12), $23.3\left(\mathrm{NHCOCH}_{3}\right), 22.3\left(\mathrm{CH}_{2}\right.$ in propargyl), 21.0 $\left(\mathrm{COCH} 3, \mathrm{C}-11\right.$ ), 19.4 (C-30), 18.3 (C-6), 17.0 (C-23, C-26), 16.2 (C-25), 14.6 (C-27). Anal. Calcd for $\mathrm{C}_{45} \mathrm{H}_{72} \mathrm{~N}_{2} \mathrm{O}_{4}: \mathrm{C}_{\text {, }}$ 76.66; $\mathrm{H}, 10.29$. Found: $\mathrm{C}, 76.71, \mathrm{H}, 10.32 \% . \mathrm{MS}: \mathrm{m} / \mathrm{z}[\mathrm{M}+\mathrm{H}]^{+}$, found $705.26\left[\mathrm{C}_{45} \mathrm{H}_{72} \mathrm{~N}_{2} \mathrm{O}_{4}\right]^{+}$requires 705.56 .

General procedure for the preparation of compounds $\mathbf{8 b}, \mathbf{9 b}, \mathbf{1 2 b - 1 5 b}$. To a solution of the appropriate ester intermediate 8a, 9a, 12a-15a (1 mmol) in $\mathrm{MeOH}(8 \mathrm{~mL})$ and THF $(4 \mathrm{~mL})$ was added $4 \mathrm{~N} \mathrm{NaOH}(4 \mathrm{~mL})$. The reaction mixture was stirred at room temperature for 15-20 h (monitoring by TLC) and then neutralized with $20 \% \mathrm{HCl}$. The solution was dried under vacuum and reconstituted with $\mathrm{CH}_{2} \mathrm{Cl}_{2}$. The organic layer was washed with brine and dried over anhydrous $\mathrm{MgSO}_{4}$ and concentrated under reduced pressure to obtain pure compounds $8 b, 9 b, 12 b-15 b$. 
$\boldsymbol{N}$-[3ß-Hydroxy-2 $\alpha$-propargyl-lup-20(29)-en-28-oyl]-N'-(tert-butoxycarbonyl)-2-ethylamine $\quad(8 \mathrm{~b})$. White powder $(0.58 \mathrm{~g}, 91 \%), \mathrm{mp} 138-140{ }^{\circ} \mathrm{C}$. $[\alpha]_{\mathrm{D}}^{23}-14.6^{\circ}$ (c $0.59, \mathrm{CH}_{2} \mathrm{Cl}_{2}$ ). IR (solution in $\mathrm{CH}_{2} \mathrm{Cl}_{2}, \mathrm{~cm}^{-1}$ ): 3366,3311 $(\mathrm{NH}), 1697,1639(\mathrm{C}=\mathrm{O}) .{ }^{1} \mathrm{H}$ NMR $\left(500 \mathrm{MHz}, \mathrm{CDCl}_{3}\right): \delta_{\mathrm{H}} 6.49,5.22$ (both br s, 2H, NH), 4.69, 4.55 (both br s, $2 \mathrm{H}$, $\mathrm{H}-29)$, 3.31-3.21 (m, 4H, $\left.-\mathrm{CH}_{2} \mathrm{NH}-,-\mathrm{CH}_{2} \mathrm{NH}-\mathrm{Boc}\right), 3.11-3.06(\mathrm{~m}, 1 \mathrm{H}, \mathrm{H}-19), 2.95(\mathrm{~d}, 1 \mathrm{H}, J 10.0 \mathrm{~Hz}, \mathrm{H}-3), 2.49-$ $0.80\left(\mathrm{~m}, 26 \mathrm{H}, \mathrm{CH}, \mathrm{CH}_{2}\right.$ in pentacyclic skeleton and propargyl), $1.64(\mathrm{~s}, 3 \mathrm{H}, \mathrm{H}-30), 1.40\left(\mathrm{~s}, 9 \mathrm{H}, \mathrm{CH}_{3}\right.$ in $\left.\mathrm{Boc}\right), 0.95$, 0.94, 0.91, 0.83, 0.75 (all s, 3H each, H-23-H-27). ${ }^{13} \mathrm{C} \mathrm{NMR} \mathrm{(125} \mathrm{MHz,} \mathrm{CDCl} 3$ ): $\delta_{\mathrm{C}} 177.3$ (C-28), 157.0 (CONHBoc), 150.9 (C-20), 109.4 (C-29), 83.1 (C in propargyl), 81.3 (C-3), 79.5 (C in Boc), 69.9 (CH in propargyl), 55.6 (C-17), 55.5 (C-5), 50.6 (C-9), 50.1 (C-18), 46.7 (C-19), 44.8 (C-1), 42.5 (C-14), 40.7 ( $\mathrm{CH}_{2}$ in ethylamine, C-8), 40.3 ( $\mathrm{CH}_{2}$ in ethylamine), 39.1 (C-4), 38.4 (C-13), 37.7 (C-22), 37.3 (C-10), 34.8 (C-7), 34.3 (C-16), 33.5 (C-2), 30.8 (C21), 29.4 (C-15), 28.4 ( $\mathrm{CH}_{3}$ in Boc, C-24), 25.6 (C-12), 22.4 ( $\mathrm{CH}_{2}$ in propargyl), 21.0 (C-11), 19.4 (C-30), 18.5 (C6), 17.0 (C-26), 16.3 (C-25), 16.2 (C-23), 14.7 (C-27). Anal. Calcd for $\mathrm{C}_{40} \mathrm{H}_{64} \mathrm{~N}_{2} \mathrm{O}_{4}$ : C, 75.43; $\mathrm{H}, 10.13$. Found: C, 75.37, $\mathrm{H}, 10.01 \%$. MS: $m / z[\mathrm{M}+\mathrm{Na}]^{+}$, found $659.45\left[\mathrm{C}_{40} \mathrm{H}_{64} \mathrm{~N}_{2} \mathrm{O}_{4}\right]^{+}$requires 659.48 .

$\boldsymbol{N}$-[3ß-Hydroxy-2 $\alpha$-propargyl-lup-20(29)-en-28-oyl]- $\boldsymbol{N}^{\prime}$-(tert-butoxycarbonyl)-8-octylamine $\quad(9 \mathrm{~b})$. White powder $(0.58 \mathrm{~g}, 80 \%), \mathrm{mp} 110-112^{\circ} \mathrm{C}$. $[\alpha]_{\mathrm{D}}^{24}-6.8^{\circ}\left(\mathrm{c} 0.24, \mathrm{CHCl}_{3}\right)$. IR (solution in $\left.\mathrm{CHCl}_{3}, \mathrm{~cm}^{-1}\right): 3366,3311(\mathrm{NH})$, 1697, 1636 (C=O). ${ }^{1} \mathrm{H}$ NMR $\left(500 \mathrm{MHz}, \mathrm{CDCl}_{3}\right): \delta_{\mathrm{H}}$ 5.64-5.66 (m, 1H, NH) $4.74(\mathrm{br} \mathrm{s}, 1 \mathrm{H}, \mathrm{H}-29), 4.59-5.54(\mathrm{~m}, 2 \mathrm{H}$, $\mathrm{H}-29, \mathrm{NH}), 3.32-3.27(\mathrm{~m}, 1 \mathrm{H}, \mathrm{H}-19), 3.19-3.10\left(\mathrm{~m}, 4 \mathrm{H},-\mathrm{CH}_{2} \mathrm{NH}-,-\mathrm{CH}_{2} \mathrm{NH}-\mathrm{Boc}\right), 2.99(\mathrm{~d}, 1 \mathrm{H}, \mathrm{J} \mathrm{10.0} \mathrm{Hz}, \mathrm{H}-3)$, 2.50-0.79 (m, 26H, CH, CH $\mathrm{CH}_{2}$ in pentacyclic skeleton, propargyl and $4 \mathrm{H}$ in octylamine), $1.68(\mathrm{~s}, 3 \mathrm{H}, \mathrm{H}-30), 1.45(\mathrm{br}$ $\mathrm{s}, 9 \mathrm{H}, \mathrm{CH}_{3}$ in Boc), 1.36-1.31 (m, 8H, $\mathrm{CH}_{2}$ in octylamine), 0.99, 0.98, 0.87, 0.80, 0.79 (all s, 3H each, $\mathrm{H}-23-\mathrm{H}-27$ ). ${ }^{13} \mathrm{C} \mathrm{NMR}\left(125 \mathrm{MHz}, \mathrm{CDCl}_{3}\right.$ ): $\delta_{\mathrm{C}} 176.0$ (C-28), 156.0 (CONH-Boc), 150.0 (C-20), 109.4 (C-29), 83.0 (C in propargyl), 81.5 (C-3), 79.1 (C in Boc), 69.9 (CH in propargyl), 55.6 (C-17), 55.5 (C-5), 50.6 (C-9), 50.2 (C-18), 46.8 (C-19), 44.9 (C-1), 44.6 (C-14), 40.8 (C-8), 40.6 ( $\mathrm{CH}_{2}$ in octylamine), 39.2 ( $\mathrm{CH}_{2}$ in octylamine), 39.1 (C-4), 38.5 (C-13), 37.8 (C-22), 37.4 (C-10), 34.8 (C-7), 34.4 (C-2), 33.9 (C-16), 30.9 (C-21), 30.0 ( $\mathrm{CH}_{2}$ in octylamine), 29.8 (C-15), 29.4 ( $\mathrm{CH}_{2}$ in octylamine), $29.2\left(\mathrm{CH}_{2}\right.$ in octylamine), 28.4 ( $\mathrm{CH}_{3}$ in $\left.\mathrm{Boc}\right), 28.3$ (C-24), 26.9 ( $\mathrm{CH}_{2}$ in octylamine), 26.7 ( $\mathrm{CH}_{2}$ in octylamine), 25.6 (C-12), 22.4 ( $\mathrm{CH}_{2}$ in propargyl), 21.0 (C-11), 19.5 (C-30), 18.5 (C-6), 17.0 (C-26), 16.2 (C-23, C-25), 14.7 (C-27). Anal. Calcd for $\mathrm{C}_{46} \mathrm{H}_{76} \mathrm{~N}_{2} \mathrm{O}_{4}$ : C, 76.62; $\mathrm{H}, 10.62$. Found: C, 76.58, H, 10.57\%. MS: $\mathrm{m} / \mathrm{z}$ $[\mathrm{M}+\mathrm{K}]^{+}$, found $759.46\left[\mathrm{C}_{46} \mathrm{H}_{76} \mathrm{~N}_{2} \mathrm{O}_{4}\right]^{+}$requires 759.54 .

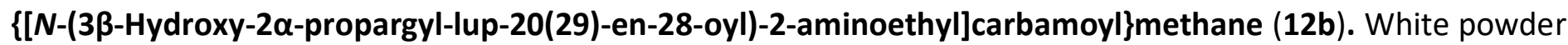
$(0.46 \mathrm{~g}, 80 \%), \mathrm{mp} 136-138^{\circ} \mathrm{C} .[\alpha]_{\mathrm{D}}^{18}-32^{\circ}\left(\mathrm{c} 0.50, \mathrm{CHCl}_{3}\right)$. IR (solution in $\left.\mathrm{CHCl}_{3}, \mathrm{~cm}^{-1}\right): 3310(\mathrm{NH}), 1637(\mathrm{C}=\mathrm{O}) .{ }^{1} \mathrm{H}$ NMR $\left(500 \mathrm{MHz}, \mathrm{CDCl}_{3}\right): \delta_{\mathrm{H}} 6.76,6.48$ (both br s, 2H, NH), 4.72, 4.59 (both br s, 2H, H-29), 3.42-3.36(m, 4H, $\left.\mathrm{CH}_{2} \mathrm{NH}-, \mathrm{CH}_{2} \mathrm{NHCOCH}_{3}\right), 3.12-3.07(\mathrm{~m}, 1 \mathrm{H}, \mathrm{H}-19), 3.00$ (d, $\left.1 \mathrm{H}, J 10.0 \mathrm{~Hz}, \mathrm{H}-3\right), 2.46-0.73\left(\mathrm{~m}, 26 \mathrm{H}, \mathrm{CH}, \mathrm{CH}_{2}\right.$ in pentacyclic skeleton and propargyl), $1.99\left(\mathrm{~s}, 3 \mathrm{H},-\mathrm{NHCOCH}_{3}\right), 1.68(\mathrm{~s}, 3 \mathrm{H}, \mathrm{H}-30), 0.99,0.98,0.93,0.82,0.78$ (all s, 3H each, H-23-H-27). ${ }^{13} \mathrm{C}$ NMR $\left(125 \mathrm{MHz}, \mathrm{CDCl}_{3}\right): \delta_{\mathrm{C}} 178.1$ (C-28), $171.4\left(\mathrm{NHCOCH}_{3}\right), 150.8$ (C-20), 109.5 (C29), 83.0 (C in propargyl), 81.4 (C-3), 70.0 ( $\mathrm{CH}$ in propargyl), 55.7 (C-17), 50.5 (C-5, C-9), 50.1 (C-18), 46.8 (C19), 44.8 (C-1), 42.5 (C-14), 40.7 (C-4, $\mathrm{CH}_{2}$ in ethylamine), 39.7 ( $\mathrm{CH}_{2}$ in ethylamine), 39.1 (C-8), 38.4 (C-13), 37.8 (C-22), 37.3 (C-10), 34.8 (C-7), 34.3 (C-2), 33.5 (C-16), 30.8 (C-21), 29.4 (C-15), 28.3 (C-24), 25.6 (C-12), 23.2 $\left(\mathrm{NHCOCH}_{3}\right), 22.4\left(\mathrm{CH}_{2}\right.$ in propargyl), 21.0 (C-11), 19.4 (C-30), 18.5 (C-6), 17.0 (C-26), 16.2 (C-23, C-25), 14.7 (C27). Anal. Calcd for $\mathrm{C}_{37} \mathrm{H}_{58} \mathrm{~N}_{2} \mathrm{O}_{3}$ : C, 76.77; $\mathrm{H}, 10.10$. Found: $\mathrm{C}, 76.82, \mathrm{H}, 10.04 \%$. MS: $\mathrm{m} / z$ [M+Na] , found 601.36 $\left[\mathrm{C}_{37} \mathrm{H}_{58} \mathrm{~N}_{2} \mathrm{O}_{3}\right]^{+}$requires 601.43 .

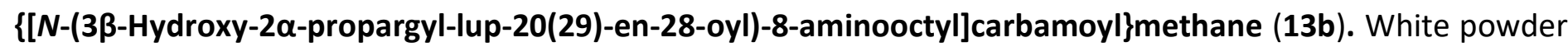
(0.59 g, 89\%), mp 112-114 ${ }^{\circ} \mathrm{C} .[\alpha]_{\mathrm{D}}^{22}-9.7^{\circ}$ ( $\mathrm{c} \mathrm{0.29,} \mathrm{CHCl}_{3}$ ). IR (solution in $\mathrm{CHCl}_{3}, \mathrm{~cm}^{-1}$ ): 3309 (NH), $1637(\mathrm{C}=\mathrm{O}) .{ }^{1} \mathrm{H}$ NMR $\left(500 \mathrm{MHz}, \mathrm{CDCl}_{3}\right)$ : $\delta_{\mathrm{H}}$ 5.79-5.70 (m, 2H, NH), 4.71, 4.57 (both br s, 2H, H-29), 3.25-3.13 (m, 5H, $-\mathrm{CH}_{2} \mathrm{NH}-$, $\left.\mathrm{CH}_{2} \mathrm{NHCOCH}_{3}, \mathrm{H}-19\right), 2.98(\mathrm{~d}, 1 \mathrm{H}, \mathrm{J} 10.0 \mathrm{~Hz}, \mathrm{H}-3), 2.45-0.80\left(\mathrm{~m}, 26 \mathrm{H}, \mathrm{CH}, \mathrm{CH}_{2}\right.$ in pentacyclic skeleton and propargyl), $1.97\left(\mathrm{~s}, 3 \mathrm{H},-\mathrm{NHCOCH}_{3}\right), 1.67(\mathrm{~s}, 3 \mathrm{H}, \mathrm{H}-30), 1.37-1.29\left(\mathrm{~m}, 12 \mathrm{H}, \mathrm{CH}_{2}\right.$ in octylamine), 0.98, 0.97, 0.93, 0.85, 0.77 (all s, 3H each, H-23-H-27). $\left.{ }^{13} \mathrm{C} \mathrm{NMR} \mathrm{(125} \mathrm{MHz,} \mathrm{CDCl} 3\right): \delta c 176.0(\mathrm{C}-28), 170.1\left(\mathrm{NHCOCH}_{3}\right), 151.0(\mathrm{C}-$ 20), 109.4 (C-29), 83.1 (C in propargyl), 81.4 (C-3), 70.0 (CH in propargyl), 55.6 (C-17), 55.5 (C-5), 50.6 (C-9), 
50.1 (C-18), 46.8 (C-19), 44.8 (C-1), 42.5 (C-14), 40.8 (C-8), 39.6 ( $\mathrm{CH}_{2}$ in octylamine), 39.1 (C-4, CH octylamine), 38.5 (C-13), 37.7 (C-22), 37.3 (C-10), 34.8 (C-7), 34.4 (C-2), 33.8 (C-16), 30.8 (C-21), 29.8 ( $\mathrm{CH}_{2}$ in octylamine), 29.5 (C-15), 29.4 ( $\mathrm{CH}_{2}$ in octylamine), 29.1 ( $\mathrm{CH}_{2}$ in octylamine), 29.1 ( $\mathrm{CH}_{2}$ in octylamine), 28.32 (C24), $26.8\left(\mathrm{CH}_{2}\right.$ in octylamine), 25.6 (C-12), $23.3\left(\mathrm{NHCOCH}_{3}\right), 22.4\left(\mathrm{CH}_{2}\right.$ in propargyl), 21.0 (C-11), 19.4 (C-30), 18.5 (C-6), 17.0 (C-26), 16.2 (C-23, C-25), 14.7 (C-27). Anal. Calcd for $\mathrm{C}_{43} \mathrm{H}_{70} \mathrm{~N}_{2} \mathrm{O}_{4}$ : C, 77.89; H, 10.64. Found: C, 77.92, $\mathrm{H}, 10.57 \%$. MS: $\mathrm{m} / \mathrm{z}[\mathrm{M}+\mathrm{H}]^{+}$, found $663.39\left[\mathrm{C}_{43} \mathrm{H}_{70} \mathrm{~N}_{2} \mathrm{O}_{4}\right]^{+}$requires 663.55 .

\section{$\mathbf{N}$-[3ß-Hydroxy-2 $\alpha$-propargyl-lup-20(29)-en-28-oyl]-N'-(tert-butoxycarbonyl)-\{3-[4-(3-aminopropyl)piperaz-} inyl]\}propylamine (14b). White powder (0.70 g, 90\%), mp 122-124 ${ }^{\circ} \mathrm{C}$. $[\alpha]_{\mathrm{D}}^{22}-8^{\circ}\left(\mathrm{c} 0.52, \mathrm{CHCl}_{3}\right)$. IR (solution in $\mathrm{CHCl}_{3}, \mathrm{~cm}^{-1}$ ): 3351, $3309(\mathrm{NH}), 1697,1638$ (C=0). ${ }^{1} \mathrm{H}$ NMR $\left(500 \mathrm{MHz}, \mathrm{CDCl}_{3}\right): \delta_{\mathrm{H}} 6.92$ (br s, 1H, NH), 5.36 (br s, $1 \mathrm{H}, \mathrm{NH}$ ), 4.71, 4.55 (both br s, 2H, H-29), 3.37-3.27, 3.17-3.10 (both $\mathrm{m}, 5 \mathrm{H},-\mathrm{CH}_{2} \mathrm{NH}-,-\mathrm{CH}_{2} \mathrm{NH}-\mathrm{Boc}, \mathrm{H}-19$ ), 2.96 (d, $1 \mathrm{H}, \mathrm{J} 10.0 \mathrm{~Hz}, \mathrm{H}-3), 2.50-2.39\left(\mathrm{~m}, 12 \mathrm{H}, \mathrm{CH}_{2}\right.$ in piperazine and propylamine), 2.29-0.70 $\left(\mathrm{m}, 26 \mathrm{H}, \mathrm{CH}, \mathrm{CH}_{2}\right.$ in pentacyclic skeleton, propargyl and $4 \mathrm{H}$ in propylamine), $1.67(\mathrm{~s}, 3 \mathrm{H}, \mathrm{H}-30), 1.41$ (br s, 9H, $\mathrm{CH}_{3}$ in $\left.\mathrm{Boc}\right), 0.96$, 0.95, 0.92, 0.84, 0.76 (all s, 3H each, $\left.\mathrm{H}-23-\mathrm{H}-27) .{ }^{13} \mathrm{C} \mathrm{NMR} \mathrm{(125} \mathrm{MHz,} \mathrm{CDCl}\right)$ : $\delta_{\mathrm{C}} 176.3(\mathrm{C}-28), 156.1$ (CONHBoc), 151.1 (C-20), 109.3 (C-29), 83.1 (C in propargyl), 81.3 (C-3), 78.9 (C in Boc), 69.9 (CH in propargyl), 57.4 ( $\mathrm{CH}_{2}$ in propylamine), $56.8\left(\mathrm{CH}_{2}\right.$ in propylamine), $55.5\left(\mathrm{CH}_{2}\right.$ in piperazine), $55.4\left(\mathrm{CH}_{2}\right.$ in piperazine $), 53.6(\mathrm{C}-17)$, 52.7 (C-5), 50.6 (C-9), 50.2 (C-18), 46.7 (C-19), 44.8 (C-1), 42.5 (C-14), 40.8 (C-8), 39.6 ( $\mathrm{CH}_{2}$ in propylamine), 39.1 (C-4), 39.0 ( $\mathrm{CH}_{2}$ in propylamine), 38.5 (C-13), 37.6 (C-22), 37.3 (C-10), 34.8 (C-7), 34.4 (C-2), 33.8 (C-16), 30.9 (C-21), 29.4 (C-15), $28.4\left(\mathrm{CH}_{3}\right.$ in Boc, C-24), $26.4\left(\mathrm{CH}_{2}\right.$ in propylamine), $25.6(\mathrm{C}-12), 25.0\left(\mathrm{CH}_{2}\right.$ in propylamine), 22.3 ( $\mathrm{CH}_{2}$ in propargyl), 21.0 (C-11), 19.4 (C-30), 18.5 (C-6), 17.0 (C-26), 16.3 (C-23, C-25), 14.7 (C-27). Anal. Calcd for $\mathrm{C}_{48} \mathrm{H}_{80} \mathrm{~N}_{4} \mathrm{O}_{4}: \mathrm{C}, 74.18 ; \mathrm{H}, 10.38$. Found: $\mathrm{C}, 74.24, \mathrm{H}, 9.84 \%$. MS: $m / z[\mathrm{M}+\mathrm{H}]^{+}$, found 777.56 $\left[\mathrm{C}_{48} \mathrm{H}_{80} \mathrm{~N}_{4} \mathrm{O}_{4}\right]^{+}$requires 777.63 .

$\boldsymbol{N}$-[3 $\beta$-Hydroxy-2 $\alpha$-propargyl-lup-20(29)-en-28-oyl]-5-piperazine-pentanoic acid (15b). White powder (0.59 g, 89\%), mp 234-236 ${ }^{\circ} \mathrm{C}$. $[\alpha]_{\mathrm{D}}^{22}-25.5^{\circ}$ (c $0.5, \mathrm{CH}_{3} \mathrm{OH}$ ). IR (solution in $\mathrm{CH}_{3} \mathrm{OH}, \mathrm{cm}^{-1}$ ): 1633 (C=O). ${ }^{1} \mathrm{H} \mathrm{NMR}(500 \mathrm{MHz}$, MeOD): $\delta_{\mathrm{H}} 4.70,4.59$ (both br s, 2H, $\left.\mathrm{H}-29\right), 3.19-3.15\left(\mathrm{~m}, 2 \mathrm{H}, \mathrm{CH}_{2}\right.$ in piperazine-pentanoic acid), 2.91-2.79 (m, $2 \mathrm{H}, \mathrm{H}-19, \mathrm{H}-13), 2.85(\mathrm{~d}, 1 \mathrm{H}, \mathrm{J} 10.0 \mathrm{~Hz}, \mathrm{H}-3), 2.47-0.75\left(\mathrm{~m}, 25 \mathrm{H}, \mathrm{CH}, \mathrm{CH}_{2}\right.$ in pentacyclic skeleton, propargyl, $12 \mathrm{H}$ in piperazine-pentanoic acid), 2.40-2.37 (m, 2H, $\left.-\mathrm{CH}_{2} \mathrm{COOH}\right), 1.65$ (s, 3H, H-30), 1.00, 0.96, 0.95, $0.88,0.75$ (all s, 3H each, H-23-H-27). ${ }^{13} \mathrm{C} N M R(125 \mathrm{MHz}, \mathrm{MeOD}): \delta_{\mathrm{c}} 179.6$ (COOH), 178.3 (C-28), 154.7 (C-20), 113.3 (C-29), 86.8 (C in propargyl), 84.8 (C-3), 73.6 ( $\mathrm{CH}$ in propargyl), 59.5 (C-5, $\mathrm{CH}_{2}$ in pentanoic acid), 58.5 (C-17), 56.4 (C18), 56.0 ( $\mathrm{CH}_{2}$ in piperazine), 54.7 (C-9), 49.6 (C-19), 48.7 (C-1), 45.8 (C-14), 44.6 (C-4), 43.0 (C-8), 41.2 (C-10), 40.9 (C-13), 39.7 (C-22), 38.6 (C-7), 38.2 (C-2), 37.2 ( $\mathrm{CH}_{2}$ in piperazine), 36.2 (C-16), 35.0 (C-21), 33.7 (C-15), 32.0 (C-24), 29.5 (C-12), 27.4 ( $\mathrm{CH}_{2}$ in pentanoic acid), 26.0 ( $\mathrm{CH}_{2}$ in propargyl), $25.9\left(\mathrm{CH}_{2}\right.$ in pentanoic acid), 25.0 (C-11), 23.1 (C-30), 22.4 (C-6), 20.8 (C-26), 20.0 (C-23), 19.8 (C-25), 18.4 (C-27). Anal. Calcd for $\mathrm{C}_{42} \mathrm{H}_{66} \mathrm{~N}_{2} \mathrm{O}_{4}: \mathrm{C}_{\text {, }}$ 76.09; $\mathrm{H}, 10.03$. Found: $\mathrm{C}, 75.94, \mathrm{H}, 9.97 \%$. MS: $\mathrm{m} / \mathrm{z}[\mathrm{M}+\mathrm{H}]^{+}$, found $663.27\left[\mathrm{C}_{42} \mathrm{H}_{66} \mathrm{~N}_{2} \mathrm{O}_{4}\right]^{+}$requires 663.51.

General procedure for the preparation of compounds 6, 16-19. To a solution of the appropriate derivative of $\mathbf{4}, \mathbf{8 b}, \mathbf{9 b}, \mathbf{1 2 b}$ or $\mathbf{1 3 b}(1 \mathrm{mmol})$ in anhydrous pyridine $(16 \mathrm{~mL})$ was added 2,2-dimethylsuccinic anhydride $(0.5$ $\mathrm{mL}, 4 \mathrm{mmol}$ ) and DMAP $(0.24 \mathrm{~g}, 2 \mathrm{mmol})$. The reaction mixture was heated under reflux for $13 \mathrm{~h}$ (monitoring by $T L C)$. Then the mixture was diluted with $\mathrm{H}_{2} \mathrm{O}(30 \mathrm{~mL})$ and extracted with EtOAc $(4 \times 30 \mathrm{~mL})$. The combined extracts were washed with brine, dried over anhydrous $\mathrm{MgSO}_{4}$, and concentrated under reduced pressure. The crude products were then chromatographed on silica gel $\left[\mathrm{CHCl}_{3}-\mathrm{MeOH}(100: 1)\right]$ to obtain pure compounds 6, 16-19.

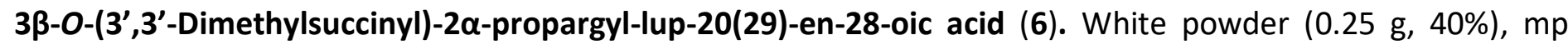
148-150 ${ }^{\circ} \mathrm{C} .[\alpha]_{\mathrm{D}}^{19}-29.8^{\circ}$ (c $0.59, \mathrm{CHCl}_{3}$ ). IR (solution in $\mathrm{CHCl}_{3}, \mathrm{~cm}^{-1}$ ): 1733, $1700(\mathrm{C}=0) .{ }^{1} \mathrm{H} \mathrm{NMR}(400 \mathrm{MHz}$, $\mathrm{CDCl}_{3}$ ): $\delta_{\mathrm{H}} 4.75,4.62$ (both br s, 2H, H-29), 4.52 (d, $1 \mathrm{H}, \mathrm{J} 10.0 \mathrm{~Hz}, \mathrm{H}-3$ ), 3.04-2.97 (m, 1H, H-19), $2.73,2.67(1 \mathrm{H}$ each, d, J $\left.15.5 \mathrm{~Hz},-\mathrm{CH}_{2}-\mathrm{CO}-\right)$, 2.21-0.80 (m, 26H, CH, CH in pentacyclic skeleton and propargyl), $1.70(\mathrm{~s}, 3 \mathrm{H}, \mathrm{H}-$ 
30), 1.35 (br s, 6H, $-\mathrm{C}\left(\mathrm{CH}_{3}\right)_{2}-$ ), 0.99, 0.96, 0.90, 0.82, 0.81 (all s, 3H each, H-23-H-27). ${ }^{13} \mathrm{C} \mathrm{NMR} \mathrm{(100} \mathrm{MHz,}$ $\mathrm{CDCl}_{3}$ ): $\delta_{\mathrm{C}} 183.4$ (COOH), 183.2 (C-28), 171.1 (- $\left.\mathrm{CH}_{2}-\mathrm{CO}-\right), 150.3$ (C-20), 109.8 (C-29), 83.4 (C-3), 82.4 (C in propargyl), 69.9 (CH in propargyl), 56.4 (C-17), 55.3 (C-5, C-9), 49.2 (C-18), 46.9 (C-19), 44.8 (C-1), $44.3\left(-\mathrm{CH}_{2}-\right.$ CO-), 42.4 (C-14), 40.7 (C-8), 40.4 (- $\left.\underline{\mathrm{C}}\left(\mathrm{CH}_{3}\right)_{2}-\right)$, 38.9 (C-4), 38.3 (C-13), 37.2 (C-22), 37.1 (C-10), 34.2 (C-7), 33.4 (C-2), 32.1 (C-16), 30.5 (C-21), 29.6 (C-15), 28.2 (C-24), $25.5\left(-\mathrm{C}\left(\mathrm{CH}_{3}\right)_{2}-\right), 25.4$ (C-12), 22.2 (CH $\mathrm{CH}_{2}$ propargyl), 20.9 (C-11), 19.3 (C-30), 18.4 (C-6), 17.2 (C-26), 17.0 (C-23), 16.2 (C-25), 14.6 (C-27). Anal. Calcd for $\mathrm{C}_{39} \mathrm{H}_{58} \mathrm{O}_{6}: \mathrm{C}_{\text {, }}$ 75.20; $\mathrm{H}, 9.39$. Found: $\mathrm{C}, 75.34, \mathrm{H}, 9.43 \% . \mathrm{MS}: \mathrm{m} / \mathrm{z}[\mathrm{M}+\mathrm{Na}]^{+}$, found $645.30\left[\mathrm{C}_{39} \mathrm{H}_{58} \mathrm{O}_{6}\right]^{+}$requires 645.41.

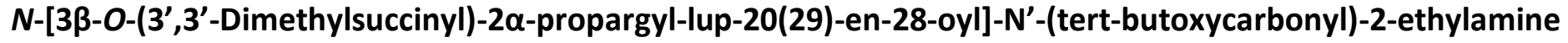

(16). White powder (0.34 g, 45\%), mp 110-112 ${ }^{\circ} \mathrm{C}$. $[\alpha]_{\mathrm{D}}^{23}-20.6^{\circ}\left(\mathrm{c} 0.47, \mathrm{CHCl}_{3}\right)$. IR (solution in $\left.\mathrm{CHCl}_{3}, \mathrm{~cm}^{-1}\right): 3369$, $3312(\mathrm{NH}), 1735,1717,1639$ (C=O). ${ }^{1} \mathrm{H}$ NMR (500 MHz, CDCl $): \delta_{\mathrm{H}} 6.41,5.10$ (both br s, 2H, NH), 4.74, 4.59 (both br s, 2H, H-29), 4.48 (d, $1 \mathrm{H}, J 10.0 \mathrm{~Hz}, \mathrm{H}-3), 3.36-3.28\left(\mathrm{~m}, 4 \mathrm{H},-\mathrm{CH}_{2} \mathrm{NH}-,-\mathrm{CH}_{2} \mathrm{NH}-\mathrm{Boc}\right), 3.15-3.11(\mathrm{~m}, 1 \mathrm{H}$, $\mathrm{H}-19)$, 2.70-2.66 (m, 2H, $\left.-\mathrm{CH}_{2}-\mathrm{CO}-\right), 2.47-0.80\left(\mathrm{~m}, 26 \mathrm{H}, \mathrm{CH}, \mathrm{CH}_{2}\right.$ in pentacyclic skeleton and propargyl), 1.69 (s, $3 \mathrm{H}, \mathrm{H}-30$ ), 1.44 (s, 9H, $\mathrm{CH}_{3}$ in $\mathrm{Boc}$ ), 1.36 (br s, 6H, $-\mathrm{C}\left(\mathrm{CH}_{3}\right)_{2}-$ ) , 0.97, 0.95, 0.88, 0.81, 0.80 (all s, 3H each, $\mathrm{H}-23-$ $\mathrm{H}-27) .{ }^{13} \mathrm{C}$ NMR (125 MHz, CDCl 3 ): $\delta_{\mathrm{C}} 181.9$ (COOH), 177.4 (C-28), 171.2 (- $\left.\mathrm{CH}_{2}-\mathrm{CO}-\right), 157.0$ (CONH-Boc), 151.0 (C-20), 109.4 (C-29), 83.4 (C-3), 82.5 (C in propargyl), 79.7 (C in Boc), 69.8 (CH in propargyl), 55.6 (C-17), 55.5 (C-5), 50.6 (C-9), 50.1 (C-18), 46.7 (C-19), 45.0 (C-1), 44.2 (- $\left.\mathrm{CH}_{2}-\mathrm{CO}-\right), 42.5$ (C-14), 40.8 (C-8), $40.6\left(\mathrm{CH}_{2}\right.$ in ethylamine), $40.3\left(\mathrm{CH}_{2}\right.$ in ethylamine, $\left.-\underline{\mathrm{C}}\left(\mathrm{CH}_{3}\right)_{2}-\right), 38.9$ (C-4), 38.4 (C-22), 37.7 (C-13), 37.2 (C-10), 34.3 (C-7), 33.5 (C-16), 33.4 (C-2), 30.8 (C-21), 29.4 (C-15), 28.4 ( $\mathrm{CH}_{3}$ in Boc), 28.1 (C-24), $25.6\left(-\mathrm{C}\left(\mathrm{CH}_{3}\right)_{2}-\right), 25.3(\mathrm{C}-12)$, 22.2 ( $\mathrm{CH}_{2}$ in propargyl), 21.0 (C-11), 19.4 (C-30), 18.3 (C-6), 17.1 (C-26), 17.0 (C-23), 16.2 (C-25), 14.7 (C-27) Anal. Calcd for $\mathrm{C}_{46} \mathrm{H}_{72} \mathrm{~N}_{2} \mathrm{O}_{7}$ : C, 72.21; H, 9.49. Found: C, 72.26, H, 9.58\%. MS: $\mathrm{m} / z$ [M+Na] ${ }^{+}$, found 787.49 $\left[\mathrm{C}_{46} \mathrm{H}_{72} \mathrm{~N}_{2} \mathrm{O}_{7}\right]^{+}$requires 787.52 .

$\mathbf{N}$-[3ß-O-(3',3'-Dimethylsuccinyl)-2 $\alpha$-propargyl-lup-20(29)-en-28-oyl]-N'-(tert-butoxycarbonyl)-8-octylamine

(17). White powder (0.76 g, 90\%), mp 90-92 ${ }^{\circ} \mathrm{C}$. $[\alpha]_{\mathrm{D}}^{21}-18.3^{\circ}\left(\mathrm{c} 0.3, \mathrm{CHCl}_{3}\right.$ ). IR (solution in $\mathrm{CHCl}_{3}, \mathrm{~cm}^{-1}$ ): 3311 (NH), 1711, 1639 (C=O). ${ }^{1} \mathrm{H}$ NMR $\left(500 \mathrm{MHz}, \mathrm{CDCl}_{3}\right): \delta_{\mathrm{H}} 5.66(\mathrm{~m}, 1 \mathrm{H}, \mathrm{NH}) 4.73(\mathrm{br} \mathrm{s}, 1 \mathrm{H}, \mathrm{H}-29), 4.58-4.60(\mathrm{~m}, 2 \mathrm{H}$, $\mathrm{H}-29, \mathrm{NH}), 4.48(\mathrm{~d}, 1 \mathrm{H}, \mathrm{J} 10.0 \mathrm{~Hz}, \mathrm{H}-3), 3.28-3.10\left(\mathrm{~m}, 5 \mathrm{H},-\mathrm{CH}_{2} \mathrm{NH}-,-\mathrm{CH}_{2} \mathrm{NH}-\mathrm{Boc}, \mathrm{H}-19\right), 2.70-2.66(\mathrm{~m}, 2 \mathrm{H},-$ $\left.\mathrm{CH}_{2}-\mathrm{CO}-\right)$, 2.47-0.79 (m, 26H, CH, CH 2 in pentacyclic skeleton, propargyl and $4 \mathrm{H}$ in octylamine), $1.68(\mathrm{~s}, 3 \mathrm{H}, \mathrm{H}-$ 30), 1.44 (br s, $9 \mathrm{H}, \mathrm{CH}_{3}$ in $\left.\mathrm{Boc}\right), 1.32-1.30\left(\mathrm{~m}, 8 \mathrm{H}, \mathrm{CH}_{2}\right.$ in octylamine and $\left.6 \mathrm{H},-\mathrm{C}\left(\mathrm{CH}_{3}\right)_{2}-\right), 0.97,0.94,0.88,0.80$, 0.79 (all s, 3H each, $\mathrm{H}-23-\mathrm{H}-27) .{ }^{13} \mathrm{C} N M R\left(125 \mathrm{MHz}, \mathrm{CDCl}_{3}\right)$ : $\delta_{\mathrm{c}} 182.6(\mathrm{COOH}), 176.3(\mathrm{C}-28), 171.3\left(-\mathrm{CH}_{2}-\mathrm{CO}_{-}\right)$, 156.0 (CONH-Boc), 151.0 (C-20), 109.4 (C-29), 83.4 (C-3), 82.4 (C in propargyl), 78.8 (C in Boc), 69.8 (CH in propargyl), 55.5 (C-17, C-5), 50.6 (C-9), 50.1 (C-18), 46.8 (C-19), 44.9 (C-1), 44.2 (- $\underline{\mathrm{CH}}_{2}-\mathrm{CO}-$ ), 42.5 (C-14), 40.8 (C-8), $40.6\left(\mathrm{CH}_{2}\right.$ in octylamine), $40.3\left(-\underline{\mathrm{C}}\left(\mathrm{CH}_{3}\right)_{2}-\right), 39.2\left(\mathrm{CH}_{2}\right.$ in octylamine), 38.9 (C-4), 38.5 (C-13), 37.7 (C-22), 37.2 (C-10), 34.3 (C-7), 33.9 (C-2), 33.4 (C-16), 30.8 (C-21), 30.0 (C-15), 29.8 ( $\mathrm{CH}_{2}$ in octylamine), $29.4\left(\mathrm{CH}_{2}\right.$ in octylamine), $29.2\left(\mathrm{CH}_{2}\right.$ in octylamine), 28.4 ( $\mathrm{CH}_{3}$ in Boc), 28.1 (C-24), $26.9\left(\mathrm{CH}_{2}\right.$ in octylamine), $26.7\left(\mathrm{CH}_{2}\right.$ in octylamine), $25.6\left(-\mathrm{C}\left(\underline{\mathrm{CH}}_{3}\right)_{2}-\right), 25.5\left(-\mathrm{C}\left(\underline{\mathrm{CH}}_{3}\right)_{2}-\right), 25.3$ (C-12), $22.2\left(\mathrm{CH}_{2}\right.$ in propargyl), 21.0 (C-11), 19.4 (C-30), 18.4 (C-6), 17.1 (C-26), 17.0 (C-23), 16.2 (C-25), 14.6 (C-27). Anal. Calcd for $\mathrm{C}_{52} \mathrm{H}_{84} \mathrm{~N}_{2} \mathrm{O}_{7}: \mathrm{C}, 73.54 ; \mathrm{H}, 9.97$. Found: $\mathrm{C}, 73.48, \mathrm{H}, 10.11 \%$. MS: $m / z[\mathrm{M}+\mathrm{K}]^{+}$, found $887.59\left[\mathrm{C}_{52} \mathrm{H}_{84} \mathrm{~N}_{2} \mathrm{O}_{7}\right]^{+}$requires 887.59 .

\{[N-(3ß-O-(3',3'-Dimethylsuccinyl)-2 $\alpha$-propargyl-lup-20(29)-en-28-oyl)-2-aminoethyl]-carbamoyl\}methane (18). White powder $(0.30 \mathrm{~g}, 42 \%)$, mp 154-156 ${ }^{\circ} \mathrm{C}$. $[\alpha]_{\mathrm{D}}^{23}-22.3^{\circ}\left(c 0.44, \mathrm{CH}_{2} \mathrm{Cl}_{2}\right.$ ). IR (solution in $\mathrm{CH}_{2} \mathrm{Cl}_{2}, \mathrm{~cm}^{-1}$ ): 3371, $3311(\mathrm{NH}), 1735,1640(\mathrm{C}=0) .{ }^{1} \mathrm{H}$ NMR $\left(500 \mathrm{MHz}, \mathrm{CDCl}_{3}\right): \delta_{\mathrm{H}} 7.11-7.08(\mathrm{~m}, 1 \mathrm{H}, \mathrm{NH}), 6.73(\mathrm{br} s, 1 \mathrm{H}, \mathrm{NH})$, 4.71, 4.58 (both br s, 2H, H-29), 4.45 (d, $1 \mathrm{H}, \mathrm{J} 10.0 \mathrm{~Hz}, \mathrm{H}-3), 3.36-3.06\left(\mathrm{~m}, 4 \mathrm{H},-\mathrm{CH}_{2} \mathrm{NH}-, \mathrm{CH}_{2} \mathrm{NHCOCH}_{3}\right), 3.10-$ $3.09(\mathrm{~m}, 1 \mathrm{H}, \mathrm{H}-19), 2.71-2.61\left(\mathrm{~m}, 2 \mathrm{H},-\mathrm{CH}_{2}-\mathrm{CO}-\right), 2.46-0.80\left(\mathrm{~m}, 26 \mathrm{H}, \mathrm{CH}, \mathrm{CH}_{2}\right.$ in pentacyclic skeleton and propargyl), 1.99 (s, 3H, $\left.-\mathrm{NHCOCH}_{3}\right), 1.67$ (s, 3H, H-30), 1.31 (br s, 6H, $\left.-\mathrm{C}\left(\mathrm{CH}_{3}\right)_{2}-\right), 0.97,0.91,0.87,0.80,0.78$ (all s, 3H each, $\mathrm{H}-23-\mathrm{H}-27) .{ }^{13} \mathrm{C} \mathrm{NMR}\left(125 \mathrm{MHz}, \mathrm{CDCl}_{3}\right)$ : $\delta_{\mathrm{c}} 180.8(\mathrm{COOH}), 177.4(\mathrm{C}-28), 171.8\left(-\mathrm{CH}_{2}-\mathrm{CO}_{-}\right), 171.4$ $\left(\mathrm{NHCOCH}_{3}\right), 151.0$ (C-20), 109.4 (C-29), 83.2 (C-3), 82.4 (C in propargyl), 69.9 (CH in propargyl), 55.6 (C-17), 55.5 (C-5), 50.6 (C-9), 50.1 (C-18), 46.7 (C-19), 44.9 (C-1), 44.2 (- $\mathrm{CH}_{2}-\mathrm{CO}-$ ), 42.5 (C-14), 40.8 (C-8), 40.6 (CH $\mathrm{CH}_{2}$ 
ethylamine), $40.2\left(\mathrm{CH}_{2}\right.$ in ethylamine, $\left.-\underline{\mathrm{C}}\left(\mathrm{CH}_{3}\right)_{2}-\right)$, 38.9 (C-4), 38.4 (C-10), 37.6 (C-13), 37.2 (C-22), 34.3 (C-7), 33.5 (C-16), 33.4 (C-2), 30.8 (C-21), 29.4 (C-15), 28.1 (C-24), $25.8\left(-\mathrm{C}\left(\underline{\mathrm{CH}}_{3}\right)_{2}-\right), 25.5\left(-\mathrm{C}\left(\underline{\mathrm{CH}}_{3}\right)_{2}-\right), 25.3$ (C-12), 23.1 $\left(\mathrm{NHCOCH}_{3}\right), 22.2\left(\mathrm{CH}_{2}\right.$ in propargyl), 21.1 (C-11), 19.4 (C-30), 18.4 (C-6), 17.1 (C-23), 17.0 (C-26), 16.2 (C-25), 14.7 (C-27). Anal. Calcd for $\mathrm{C}_{43} \mathrm{H}_{66} \mathrm{~N}_{2} \mathrm{O}_{6}: \mathrm{C}, 73.05 ; \mathrm{H}, 9.41$. Found: C, 73.10, H, 9.38\%. MS: $m / z$ [M+Na] ${ }^{+}$, found $729.49\left[\mathrm{C}_{43} \mathrm{H}_{66} \mathrm{~N}_{2} \mathrm{O}_{6}\right]^{+}$requires 729.48 .

\{[N-(3ß-O-(3',3'-Dimethylsuccinyl)-2 $\alpha$-propargyl-lup-20(29)-en-28-oyl)-8-aminooctyl]-carbamoyl\}methane (19). White powder $(0.39 \mathrm{~g}, 49 \%), \mathrm{mp} 112-114^{\circ} \mathrm{C}$. $[\alpha]_{\mathrm{D}}^{22}-22.2^{\circ}\left(\mathrm{c} 0.36, \mathrm{CHCl}_{3}\right.$ ). IR (solution in $\left.\mathrm{CHCl}_{3}, \mathrm{~cm}^{-1}\right): 3308$ (NH), $1733(\mathrm{C}=\mathrm{O}) .{ }^{1} \mathrm{H}$ NMR $\left(500 \mathrm{MHz}, \mathrm{CDCl}_{3}\right): \delta_{\mathrm{H}}$ 5.87-5.72 (m, 2H, NH), 4.72, 4.58 (both br s, 2H, H-29), 4.47 (d, $1 \mathrm{H}, \mathrm{J} 10.0 \mathrm{~Hz}, \mathrm{H}-3), 3.22-3.14\left(\mathrm{~m}, 5 \mathrm{H},-\mathrm{CH}_{2} \mathrm{NH}-, \mathrm{CH}_{2} \mathrm{NHCOCH}_{3}, \mathrm{H}-19\right), 2.70-2.59\left(\mathrm{~m}, 2 \mathrm{H},-\mathrm{CH}_{2}-\mathrm{CO}-\right), 2.49-0.80$ ( $\mathrm{m}, 26 \mathrm{H}, \mathrm{CH}, \mathrm{CH}_{2}$ in pentacyclic skeleton and propargyl), $1.94\left(\mathrm{~s}, 3 \mathrm{H},-\mathrm{NHCOCH}_{3}\right), 1.67(\mathrm{~s}, 3 \mathrm{H}, \mathrm{H}-30), 1.55-1.25$ (m, $18 \mathrm{H}, \mathrm{CH}_{2}$ in octylamine and $\mathrm{CH}_{3}$ in $-\mathrm{C}\left(\mathrm{CH}_{3}\right)_{2}-$ ), 0.96, 0.93, 0.87, 0.80, 0.79 (all s, 3H each, $\mathrm{H}-23-\mathrm{H}-27$ ). ${ }^{13} \mathrm{C}$ NMR (125 MHz, CDCl 3 ): $\delta_{c} 181.9$ (COOH), 176.1 (C-28), $171.2\left(-\mathrm{CH}_{2}-\underline{\mathrm{CO}}-\right)$, 170.7 ( $\left.\mathrm{NH}_{\mathrm{COCH}}\right), 151.0$ (C-20), 109.4 (C-29), 83.3 (C-3), 82.5 (C in propargyl), 69.8 (CH in propargyl), 55.5 (C-17, C-5), 50.6 (C-9), 50.1 (C-18), 46.8 (C-19), 44.9 (C-1), 44.2 (- $\left.\mathrm{CH}_{2}-\mathrm{CO}-\right)$, 42.5 (C-14), 40.8 (C-8), 40.3 (- $\left.\mathrm{C}\left(\mathrm{CH}_{3}\right)_{2}-\right), 39.7$ ( $\mathrm{CH}_{2}$ in octylamine), 39.2 ( $\mathrm{CH}_{2}$ in octylamine), 38.9 (C-4), 38.5 (C-13), 37.7 (C-10), 37.2 (C-22), 34.3 (C-7), 33.8 (C-2), 33.4 (C-16), 30.8 (C21), 29.8 (C-15), 29.7 ( $\mathrm{CH}_{2}$ in octylamine), 29.4 ( $\mathrm{CH}_{2}$ in octylamine), 29.1( $\mathrm{CH}_{2}$ in octylamine), 28.1 (C-24), 26.8 $\left(\mathrm{CH}_{2}\right.$ in octylamine), $25.6\left(-\mathrm{C}\left(\underline{\mathrm{CH}}_{3}\right)_{2}-\right), 25.5\left(-\mathrm{C}\left(\underline{\mathrm{CH}}_{3}\right)_{2}-\right), 25.4(\mathrm{C}-12), 23.3\left(\mathrm{NHCOCH}_{3}\right), 22.2\left(\mathrm{CH}_{2}\right.$ in propargyl), 21.0 (C-11), 19.4 (C-30), 18.4 (C-6), 17.1 (C-26), 17.0 (C-23), 16.2 (C-25), 14.6 (C-27). Anal. Calcd for $\mathrm{C}_{49} \mathrm{H}_{78} \mathrm{~N}_{2} \mathrm{O}_{6}$ : C, 74.39; $\mathrm{H}, 9.94$. Found: $\mathrm{C}, 74.43, \mathrm{H}, 10.06 \% . \mathrm{MS}: \mathrm{m} / z[\mathrm{M}+\mathrm{Na}]^{+}$, found $813.36\left[\mathrm{C}_{49} \mathrm{H}_{78} \mathrm{~N}_{2} \mathrm{O}_{6}\right]^{+}$requires 813.58 .

General procedure for click reactions. A. To a solution of alkyne 4 or 5 ( $1 \mathrm{mmol})$ in $\mathrm{Bu}^{\mathrm{t}} \mathrm{OH}(15 \mathrm{~mL})$ were added azidothymidine $(0.21 \mathrm{~g}, 0.8 \mathrm{mmol})$, $\mathrm{Cul}(0.04 \mathrm{~g}, 0.2 \mathrm{mmol})$ and stirring of the reaction mixture at $70{ }^{\circ} \mathrm{C}$ for $12 \mathrm{~h}$ (monitoring by TLC). Then the mixture was chromatographed on silica gel $\left[\mathrm{CHCl}_{3}-\mathrm{MeOH}(100: 1 \rightarrow 1: 1)\right]$ to obtain pure compounds 20 and 21.

B. To a solution of the alkyne 4-6, 12b, 14b, 15b, 16-19 (1 mmol) in DMSO (2 mL) were added azidothymidine $(0.29 \mathrm{~g}, 1.1 \mathrm{mmol})$, sodium ascorbate $(0.20 \mathrm{~g}, 1.01 \mathrm{mmol}), \mathrm{CuSO}_{4} \cdot 5 \mathrm{H}_{2} \mathrm{O}(0.25 \mathrm{~g}, 1.01 \mathrm{mmol})$ and stirring of the reaction mixture was continued at room temperature for $2 \mathrm{~h}$ (monitoring by TLC). Then the mixture was chromatographed on silica gel $\left[\mathrm{CHCl}_{3}-\mathrm{MeOH}(100: 1 \rightarrow 1: 1)\right]$ to obtain pure compounds 20-29.

[1-(3'-Deoxythymidine)-1H-1,2,3-triazol-4-yl]-2 $\boldsymbol{\alpha}$-methyl-3ß-hydroxy-lup-20(29)-en-28-oic acid (20). White $\operatorname{powder}\left(\mathrm{A}, 0.53 \mathrm{~g}, 70 \% ; \mathrm{B}, 0.54 \mathrm{~g}, 71 \%\right.$ ), mp 206-208 ${ }^{\circ} \mathrm{C}$. $[\alpha]_{\mathrm{D}}^{18}-25.3^{\circ}\left(c 0.55, \mathrm{CH}_{3} \mathrm{OH}\right.$ ). IR (solution in $\mathrm{CH}_{3} \mathrm{OH}, \mathrm{cm}^{-}$ $\left.{ }^{1}\right): 1682(\mathrm{C}=0) .{ }^{1} \mathrm{H}$ NMR $(500 \mathrm{MHz}, \mathrm{MeOD}): \delta_{\mathrm{H}} 7.93$ (s, 1H, H-Thy), $7.88(\mathrm{~s}, 1 \mathrm{H}, \mathrm{H}$-triazol), $6.49(\mathrm{t}, 1 \mathrm{H}, J 5.0 \mathrm{~Hz}, \mathrm{H}-$ 1'-ribose), 5.44-5.41 (m, 1H, H-3'-ribose), 4.71, 4.59 (both br s, 2H, H-29), 4.37-4.36 (m, 1H, H-4'-ribose), 3.93 and 3.79 (1H each, dd, J 10.0, $5.0 \mathrm{~Hz}, \mathrm{H}-5^{\prime}$-ribose), 3.19 (d, 1H, J $\left.15.0 \mathrm{~Hz},=\mathrm{C}-\mathrm{CH}_{2}-\right), 3.02-2.97\left(\mathrm{~m}, 2 \mathrm{H}, \mathrm{H}-19, \mathrm{H}-2^{\prime}-\right.$ ribose), $2.83\left(\mathrm{~d}, 1 \mathrm{H}, J 10 \mathrm{~Hz}, \mathrm{H}-3\right.$ ), 2.77-2.74 (m, 1H, H-2'-ribose), 2.52 (dd, 1H, J 15.0, $10.0 \mathrm{~Hz},=\mathrm{C}-\mathrm{CH}_{2^{-}}$), 2.29$0.60\left(\mathrm{~m}, 23 \mathrm{H}, \mathrm{CH}, \mathrm{CH}_{2}\right.$ in pentacyclic skeleton), 1.92 (s, 3H, $\left.\mathrm{CH}_{3}-\mathrm{Thy}\right), 1.70$ (s, 3H, H-30), 0.99, 0.98, 0.95, 0.81, 0.80 (all s, 3H each, H-23-H-27). ${ }^{13} \mathrm{C}$ NMR (125 MHz, MeOD): $\delta_{c} 180.5$ (C-28), 166.5, 152.4 (C=0-Thy), 152.1 (C20), 148.1 (C-triazol), 138.4 (CH-Thy), 123.9 (CH-triazol ), 111.8 (C-Thy), 110.4 (C-29), 86.8 (1'-ribose), 86.5 (4'ribose), 83.1 (C-3), 62.2 (5'-ribose), 60.9 (3'-ribose), 57.6 (C-17), 57.1 (C-5), 52.0 (C-9), 50.0 (C-18), 49.0 (C-19), 46.4 (C-1), 43.7 (C-14), 42.1 (C-8), 40.6 (C-10), 39.7 (C-13), 39.1 (2'-ribose), 38.5 (C-4), 38.3 (C-22), 37.3 (C-2), 35.7 (C-7), 33.5 (C-16), 31.9 (C-21), 31.0 (C-15), 30.0 (=C- $\mathrm{CH}_{2}-$ ), 29.2 (C-24), 27.0 (C-12), 22.2 (C-11), 19.8 (C-6, C-30), 17.9 (C-26), 17.7 ( $\left.\mathrm{CH}_{3}-\mathrm{Thy}\right), 16.8$ (C-23, C-25), 15.4 (C-27). Anal. Calcd for $\mathrm{C}_{43} \mathrm{H}_{63} \mathrm{~N}_{5} \mathrm{O}_{7}: \mathrm{C}, 67.78 ; \mathrm{H}, 8.33$. Found: $\mathrm{C}, 68.32, \mathrm{H}, 8.28 \%$. MS: $m / z[\mathrm{M}+\mathrm{Na}]^{+}$, found $784.33\left[\mathrm{C}_{43} \mathrm{H}_{63} \mathrm{~N}_{5} \mathrm{O}_{7}\right]^{+}$requires 784.46 .

[1-(3'-Deoxythymidine)-1H-1,2,3-triazol-4-yl]-2 $\alpha$-methyl-3ß-acetoxylup-20(29)-en-28-oic acid (21). White powder (A, $0.44 \mathrm{~g}, 55 \%, \mathrm{~B}, 0.55 \mathrm{~g}, 68 \%)$, mp 196-198 ${ }^{\circ} \mathrm{C}$. [ $\left.\alpha\right]_{\mathrm{D}}^{22}-14^{\circ}\left(\mathrm{c} 0.65, \mathrm{CH}_{3} \mathrm{OH}\right.$ ). IR (solution in $\mathrm{CH}_{3} \mathrm{OH}, \mathrm{cm}^{-1}$ ): 1696 (C=O). ${ }^{1} \mathrm{H}$ NMR (500 MHz, MeOD): $\delta_{H} 7.93$ (s, 1H, H-Thy), 7.90 (s, 1H, H-triazol), 6.48 (t, 1H, J 5.0 Hz, H-1'- 
ribose), 5.44-5.40 (m, 1H, H-3'-ribose), 4.71, 4.60 (both br s, 2H, H-29), 2.52 (d, $1 \mathrm{H}, J 10.0 \mathrm{~Hz}, \mathrm{H}-3$ ), 4.38-4.36 ( $\mathrm{m}, 1 \mathrm{H}, \mathrm{H}-4^{\prime}$-ribose), 3.92 and 3.80 (1H each, dd, J 10.0, 5.0 Hz, H-5'-ribose), 3.04-2.99 (m, 1H, H-19), 2.95-2.90 ( $\mathrm{m}, 1 \mathrm{H}, \mathrm{H}-2^{\prime}$-ribose), 2.76-2.68 (m, 2H, H-2'-ribose, =C- $\mathrm{CH}_{2}-$ ), 2.52 (dd, $1 \mathrm{H}, J 15.0,10.0 \mathrm{~Hz},=\mathrm{C}-\mathrm{CH}_{2}-$ ), 2.27-0.60 (m, 23H, CH, CH $\mathrm{CH}_{2}$ in pentacyclic skeleton), 2.07 (s, 3H, $\left.\mathrm{CH}_{3}-\mathrm{Thy}\right), 1.93\left(\mathrm{~s}, 3 \mathrm{H}, \mathrm{COCH}_{3}\right), 1.72(\mathrm{~s}, 3 \mathrm{H}, \mathrm{H}-30), 1.03$, 0.96, 0.90, 0.86, 0.84 (all s, 3H each, H-23-H-27). ${ }^{13} \mathrm{C}$ NMR (125 MHz, MeOD): $\delta_{\mathrm{C}} 178.5$ (C-28), $171.8\left(\mathrm{COCH}_{3}\right)$, 165.0 (C=O-Thy), 150.9 (C-20), 150.5 (C=O-Thy), 146.0 (C-triazol), 136.9 (CH-Thy), 122.4 (CH-triazol), 110.2 (CThy), 108.9 (C-29), 85.3 (1'-ribose), 85.0 (4'-ribose), 83.8 (C-3), 60.7 (5'-ribose), 59.4 (3'-ribose), 56.0 (C-17), 55.4 (C-5), 50.4 (C-9), 49.2 (C-18), 47.3 (C-19), 44.8 (C-1), 42.2 (C-14), 40.6 (C-4), 38.7 (C-8), 38.2 (C-13), 37.6 (2'-ribose), 37.0 (C-22), 36.7 (C-10), 34.0 (C-2), 31.9 (C-7), 30.3 (C-16), 29.4 (C-15, C-21), 28.4 (=C- $\left.\mathrm{CH}_{2}-\right), 27.4$ (C24), 25.4 (C-12), 20.7 (C-11), $19.6\left(\mathrm{COCH}_{3}\right), 18.2$ (C-30), 18.1 (C-6), 16.2 (C-26), 16.1 (C-23), 15.2 (C-25), 13.8 (C27), 11.1 ( $\left.\mathrm{CH}_{3}-\mathrm{Thy}\right)$. Anal. Calcd for $\mathrm{C}_{45} \mathrm{H}_{65} \mathrm{~N}_{5} \mathrm{O}_{8}$ : C, 67.22; $\mathrm{H}, 8.15$. Found: $\mathrm{C}, 67.34, \mathrm{H}, 8.24 \%$. MS: $m / z$ [M+Na] ${ }^{+}$, found $826.27\left[\mathrm{C}_{45} \mathrm{H}_{65} \mathrm{~N}_{5} \mathrm{O}_{8}\right]^{+}$requires 826.47.

[1-(3'-Deoxythymidine)-1H-1,2,3-triazol-4-yl]-2 $\alpha$-methyl-3ß-O-(3',3'-dimethylsuccinyl)lup-20(29)-en-28-oic acid (22). White powder (B, $0.56 \mathrm{~g}, 63 \%$ ), mp 270-272 ${ }^{\circ} \mathrm{C}$. $[\alpha]_{\mathrm{D}}^{18}-1^{\circ}\left(c 0.28, \mathrm{CH}_{3} \mathrm{OH}\right.$ ). IR (solution in $\mathrm{CH}_{3} \mathrm{OH}, \mathrm{cm}^{-}$ ${ }^{1}$ ): $1697(\mathrm{C}=\mathrm{O}) .{ }^{1} \mathrm{H}$ NMR $(500 \mathrm{MHz}, \mathrm{MeOD}): \delta_{\mathrm{H}} 7.66(\mathrm{~s}, 1 \mathrm{H}, \mathrm{H}$-Thy), 7.45 (s, 1H, H-triazol), 6.25 (t, $1 \mathrm{H}, J 5.0 \mathrm{~Hz}, \mathrm{H}-$ 1'-ribose), 5.17-5.15 (m, 1H, H-3'-ribose), 4.47, 4.35 (both br s, 2H, H-29), 4.52 (d, 1H, J $10.0 \mathrm{~Hz}, \mathrm{H}-3$ ), 4.15-4.13 (m, $1 \mathrm{H}, \mathrm{H}-4^{\prime}$-ribose), 3.74 and 3.60 (1H each, dd, J 10.0, $5.0 \mathrm{~Hz}, \mathrm{H}-5^{\prime}$-ribose), 2.78-2.74 (m, 2H, H-19, H-2'ribose), 2.35 and 2.52 (1H each, d, J $15.0 \mathrm{~Hz},-\mathrm{CH}_{2}-\mathrm{CO}-$ ), 2.53-2.30 (m, 3H, H-2'-ribose, =C-CH2-), 2.03-0.50 (m, $23 \mathrm{H}, \mathrm{CH}, \mathrm{CH}_{2}$ in pentacyclic skeleton), 1.71 (s, 3H, $\left.\mathrm{CH}_{3}-\mathrm{Thy}\right), 1.45(\mathrm{~s}, 3 \mathrm{H}, \mathrm{H}-30), 1.04$ (br s, 6H, $\left.-\mathrm{C}\left(\mathrm{CH}_{3}\right)_{2}-\right), 0.75$, 0.71, 0.63, 0.62, 0.58 (all s, 3H each, H-23-H-27). ${ }^{13} \mathrm{C}$ NMR (125 MHz, MeOD): $\delta c 182.8$ (COOH), 179.7 (C-28), 172.7 (- $\mathrm{CH}_{2}$ - $\mathrm{CO}-$ ), 164.5 (C=O-Thy), 150.6 (C-20), 150.3 (C=O-Thy), 145.7 (C-triazol), 136.5 (CH-Thy), 121.9 (CHtriazol), 110.7 (C-Thy), 108.9 (C-29), 85.3 (1'-ribose), 84.7 (4'-ribose), 83.6 (C-3), 60.4 (5'-ribose), 58.9 (3'ribose), 55.9 (C-5), 55.0 (C-17), 50.0 (C-9, C-18), 46.6 (C-19), 44.7 (C-1), 44.3 (- $\left.\mathrm{CH}_{2}-\mathrm{CO}-\right), 42.0$ (C-14), 40.3 ($\left.\mathrm{C}\left(\mathrm{CH}_{3}\right)_{2}-\right)$, 38.6 (C-4), 37.8 (C-13), 37.6 (2'-ribose), 36.8 (C-8, C-22), 33.7 (C-10), 33.4 (C-2), 31.9 (C-7), 30.1 (C16), 29.2 (C-15, C-21), 28.1 (=C- $\left.\underline{\mathrm{CH}}_{2}-\right), 27.7$ (C-24), $25.7\left(-\mathrm{C}\left(\underline{\mathrm{CH}}_{3}\right)_{2}-\right), 25.0(\mathrm{C}-12), 25.0\left(-\mathrm{C}\left(\mathrm{CH}_{3}\right)_{2}-\right), 20.5$ (C-11), 18.6 (C-30), 17.9 (C-6), 16.6 (C-26), 16.3 (C-23), 15.4 (C-25), 14.1 (C-27), 11.6 ( $\mathrm{CH}_{3}$-Thy). Anal. Calcd for $\mathrm{C}_{49} \mathrm{H}_{71} \mathrm{~N}_{5} \mathrm{O}_{10}: \mathrm{C}, 66.12 ; \mathrm{H}, 8.04$. Found: $\mathrm{C}, 66.23, \mathrm{H}, 8.12 \%$. MS: $\mathrm{m} / z[\mathrm{M}+\mathrm{Na}]^{+}$, found $912.49\left[\mathrm{C}_{49} \mathrm{H}_{71} \mathrm{~N}_{5} \mathrm{O}_{10}\right]^{+}$ requires 912.51.

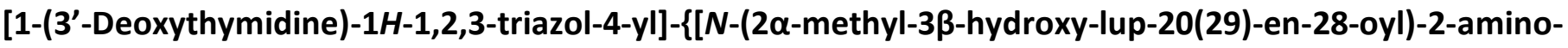

ethyl]carbamoyl\}methane (23). White powder (B, $0.57 \mathrm{~g}, 67 \%), \mathrm{mp} 192-194{ }^{\circ} \mathrm{C}$. $[\alpha]_{\mathrm{D}}^{22}-23.3^{\circ}\left(c 0.54, \mathrm{CH}_{3} \mathrm{OH}\right)$. IR (solution in $\mathrm{CH}_{3} \mathrm{OH}, \mathrm{cm}^{-1}$ ): $3311(\mathrm{NH}), 1636$ (C=O). ${ }^{1} \mathrm{H}$ NMR (500 MHz, MeOD): $\delta_{\mathrm{H}} 7.94$ (s, 1H, H-Thy), 7.88 (s, $1 \mathrm{H}, \mathrm{H}$-triazol), 7.62 (br s, $1 \mathrm{H}, \mathrm{NH}), 6.50$ (t, 1H, J $5.0 \mathrm{~Hz}, \mathrm{H}$-1'-ribose), 5.44-5.42 (m, 1H, H-3'-ribose), 4.87, 4.70 (both br s, 2H, H-29), 4.37-4.36 (m, 1H, H-4'-ribose), 3.93 and 3.79 (1H each, dd, J 10.0, $5.0 \mathrm{~Hz}, \mathrm{H}-5^{\prime}$-ribose), 3.88-3.28 (m, 4H, $\left.-\mathrm{CH}_{2} \mathrm{NH}-, \mathrm{CH}_{2} \mathrm{NHCOCH}_{3}\right), 3.19\left(\mathrm{~d}, 1 \mathrm{H}, \mathrm{J} 15.0 \mathrm{~Hz},=\mathrm{C}-\mathrm{CH}_{2}-\right), 3.10-3.06(\mathrm{~m}, 1 \mathrm{H}, \mathrm{H}-19), 2.98-2.93$ (m, 1H, H-2'-ribose), 2.82 (d, 1H, J $10.0 \mathrm{~Hz}, \mathrm{H}-3), 2.79-2.74\left(\mathrm{~m}, 1 \mathrm{H}, \mathrm{H}-2^{\prime}\right.$-ribose), 2.54-2.49 (m, 1H, =C-CH $\left.2^{-}\right)$, 2.12-0.59 (m, 23H, CH, CH 2 in pentacyclic skeleton and $1 \mathrm{H}, \mathrm{NH}), 1.95\left(\mathrm{~s}, 3 \mathrm{H},-\mathrm{NHCOCH}_{3}\right), 1.92\left(\mathrm{~s}, 3 \mathrm{H}, \mathrm{CH}_{3}-\mathrm{Thy}\right)$, 1.69 (s, 3H, H-30), 0.99, 0.98, 0.94, 0.81, 0.80 (all s, 3H each, H-23-H-27). ${ }^{13} \mathrm{C} \mathrm{NMR} \mathrm{(125} \mathrm{MHz,} \mathrm{MeOD):} \delta_{c} 179.7$ (C-28), $173.8\left(\mathrm{NHCOCH}_{3}\right), 166.5$ (C=O-Thy), 152.4 (C-20, C=O-Thy), 148.1 (C-triazol), 138.4 (CH-Thy), 123.8 (CHtriazol ), 111.8 (C-Thy), 110.2 (C-29), 86.8 (1'-ribose), 86.5 (4'-ribose), 83.1 (C-3), 62.2 (5'-ribose), 60.9 (3'ribose), 57.1 (C-5, C-17), 52.1 (C-9), 51.5 (C-18), 48.6 (C-19), 46.4 (C-1), 43.7 (C-14), 42.1 (C-8), 40.5 (C-4), 40.4 ( $\mathrm{CH}_{2}$ in ethylamine), 40.3 ( $\mathrm{CH}_{2}$ in ethylamine), 39.4 (C-13), 39.1 (2'-ribose), 39.0 (C-22), 38.5 (C-10), 37.3 (C-2), 35.7 (C-7), 34.2 (C-16), 32.0 (C-21), 30.7 (C-15), 29.9 (=C- $\underline{\mathrm{CH}}_{2}-$ ), 29.2 (C-24), 27.1 (C-12), 22.9 ( $\left.\mathrm{NHCO}^{-\mathrm{CH}_{3}}\right), 22.2$ (C-11), 19.8 (C-6, C-30), 17.6 (C-26), 17.1 (C-23), 16.9 (C-25), 15.3 (C-27), 12.7 ( $\mathrm{CH}_{3}$-Thy). Anal. Calcd for $\mathrm{C}_{47} \mathrm{H}_{71} \mathrm{~N}_{7} \mathrm{O}_{7}: \mathrm{C}, 66.72 ; \mathrm{H}, 8.46$. Found: $\mathrm{C}, 66.65, \mathrm{H}, 8.51 \% . \mathrm{MS}: \mathrm{m} / z[\mathrm{M}+\mathrm{K}]^{+}$, found $884.36\left[\mathrm{C}_{47} \mathrm{H}_{71} \mathrm{~N}_{7} \mathrm{O}_{7}\right]^{+}$requires 884.51 . 
\{[1-(3'-Deoxythymidine)-1H-1,2,3-triazol-4-yl]-N-[N'-(tert-butoxycarbonyl)-\{3-[4-(3-aminopropyl)piperazinyl]propyl\}\}-2 $\alpha$-methyl betulinic acid amide (24). White powder (B, $0.75 \mathrm{~g}, 72 \%), \mathrm{mp} 142-144^{\circ} \mathrm{C} .[\alpha]_{\mathrm{D}}^{22}-13^{\circ}(c$ 0.63, $\mathrm{CH}_{3} \mathrm{OH}$ ). IR (solution in $\left.\mathrm{CH}_{3} \mathrm{OH}, \mathrm{cm}^{-1}\right)$ : $3310(\mathrm{NH}), 1637(\mathrm{C}=0) .{ }^{1} \mathrm{H}$ NMR $(400 \mathrm{MHz}, \mathrm{MeOD}): \delta_{\mathrm{H}} 7.93(\mathrm{~s}, 1 \mathrm{H}$, H-Thy), 7.87 (s, 1H, H-triazole), 6.49 (t, 1H, J 5.0 Hz, H-1'-ribose), 5.44-5.41 (m, 1H, H-3'-ribose), 4.71, 4.59 (both br s, 2H, H-29), 4.39-4.34 (m, 1H, H-4'-ribose), 3.93 and 3.78 (1H each, dd, J 10.0, 5.0 Hz, H-5'-ribose), 3.29-2.93 (m, 7H, $-\mathrm{CH}_{2} \mathrm{NH}-,-\mathrm{CH}_{2} \mathrm{NH}-\mathrm{Boc}, \mathrm{H}-19, \mathrm{H}-2^{\prime}$-ribose, =C-CH $\left.2^{-}\right), 2.82(\mathrm{~d}, 1 \mathrm{H}, \mathrm{J} 12.0 \mathrm{~Hz}, \mathrm{H}-3), 2.79-2.36$ (m, $14 \mathrm{H}, \mathrm{CH}_{2}$ in piperazine and propylamine, $\mathrm{H}-2^{\prime}$-ribose, $\left.=\mathrm{C}_{-} \mathrm{CH}_{2}-\right), 2.14-0.58\left(\mathrm{~m}, 23 \mathrm{H}, \mathrm{CH}, \mathrm{CH}_{2}\right.$ in pentacyclic skeleton, $4 \mathrm{H}$ in propylamine and $2 \mathrm{H}, \mathrm{NH}), 1.93\left(\mathrm{~s}, 3 \mathrm{H}, \mathrm{CH}_{3}-\mathrm{Thy}\right), 1.70(\mathrm{~s}, 3 \mathrm{H}, \mathrm{H}-30), 1.45$ (br s, 9H, $\mathrm{CH}_{3}$ in $\mathrm{Boc}$ ), 0.99, 0.98, 0.95, 0.83, 0.81 (all s, 3H each, $\mathrm{H}-23-\mathrm{H}-27) .{ }^{13} \mathrm{C} N M R(100 \mathrm{MHz}, \mathrm{MeOD}): \delta_{\mathrm{C}} 179.0$ (C-28), 166.4 (C=O-Thy), 158.5 (CONH-Boc), 152.3 (C-20, C=O-Thy), 148.0 (C-triazole), 138.3 (CH-Thy), 123.8 (CH-triazole), 111.7 (C-Thy), 110.2 (C-29), 86.7 (1'-ribose), 86.5 (4'-ribose), 83.0 (C-3), 79.9 (C in Boc), 62.5 (5'-ribose), 60.9 (3'-ribose), $57.4\left(\mathrm{CH}_{2}\right.$ in propylamine), 57.0 (C-5, C-17), $56.8\left(\mathrm{CH}_{2}\right.$ in propylamine), 55.8, $53.7\left(\mathrm{CH}_{2}\right.$ in piperazine), 52.1 (C-9), 51.4 (C-18), 48.6 (C-19), 46.4 (C-1), 43.6 (C-14), 42.1 (C-8), 40.5 (C-10), 39.7 (C-13), 39.5 ( $\mathrm{CH}_{2}$ in propylamine), 39.1 (2'-ribose), 38.9 ( $\mathrm{CH}_{2}$ in propylamine), 38.6 (C-4), 38.4 (C-22), 37.3 (C-2), 35.7 (C-7), 34.3 (C-16), 32.0 (C-21), 30.6 (C-15), 29.9 (=C- $\underline{\mathrm{CH}}_{2}-$ ), 29.2 (C-24), 29.0 ( $\mathrm{CH}_{3}$ in Boc), 27.7 ( $\mathrm{CH}_{2}$ in propylamine), $27.4\left(\mathrm{CH}_{2}\right.$ in propylamine), 27.0 (C-12), 22.2 (C-11), 19.9 (C-6, C-30), 17.6 (C-26), 17.2 (C-23), 17.1 (C-25), 15.3 (C-27), $11.3\left(\mathrm{CH}_{3}\right.$-Thy). Anal. Calcd for $\mathrm{C}_{58} \mathrm{H}_{93} \mathrm{~N}_{9} \mathrm{O}_{8}: \mathrm{C}, 66.70 ; \mathrm{H}, 8.98$. Found: C, 66.67, $\mathrm{H}, 8.87 \% . \mathrm{MS}: \mathrm{m} / \mathrm{z}$ $[\mathrm{M}+\mathrm{H}]^{+}$, found $1044.65\left[\mathrm{C}_{58} \mathrm{H}_{93} \mathrm{~N}_{9} \mathrm{O}_{8}\right]^{+}$requires 1044.72 .

\{[1-(3'-Deoxythymidine)-1H-1,2,3-triazol-4-yl]-[N-2 $\alpha$-methyl-3 $\beta$-hydroxy-lup-20(29)-en-28-oyl]\}-5-piperazinylpentanoic acid (25). White powder (B, $0.56 \mathrm{~g}, 60 \%), \mathrm{mp} 242-244{ }^{\circ} \mathrm{C}$. $[\alpha]_{\mathrm{D}}^{18}-12^{\circ}(\mathrm{c} 0.55$, DMSO). IR (solution in $\mathrm{CH}_{3} \mathrm{OH}, \mathrm{cm}^{-1}$ ): 1636 (C=O). ${ }^{1} \mathrm{H}$ NMR (500 MHz, MeOD): $\delta_{\mathrm{H}} 7.93$ (br s, 2H, H-Thy, H-triazol), 6.50 (t, $1 \mathrm{H}, J 5.0 \mathrm{~Hz}$, $\mathrm{H}-1^{\prime}$-ribose), 5.55-5.52 (m, 1H, H-3'-ribose), 4.70, 4.60 (both br s, 2H, H-29), 4.39-4.34 (m, 1H, H-4'-ribose), $3.92\left(1 \mathrm{H}, \mathrm{dd}, J 10.0,5.0 \mathrm{~Hz}, \mathrm{H}-5^{\prime}\right.$-ribose), 3.79-3.75 (m, 5H, H-5'-ribose and $\mathrm{CH}_{2}$ in piperazine), 3.10-3.06 (m, $1 \mathrm{H}, \mathrm{H}-19), 3.00-2.68\left(\mathrm{~m}, 12 \mathrm{H}, \mathrm{H}-3, \mathrm{H}-13, \mathrm{H}-2^{\prime}\right.$-ribose, $=\mathrm{C}-\mathrm{CH}_{2^{-}}, \mathrm{CH}_{2}$ in piperazine and pentanoic acid), 2.19-0.78 ( $\mathrm{m}, 22 \mathrm{H}, \mathrm{CH}, \mathrm{CH}_{2}$ in pentacyclic skeleton and $6 \mathrm{H}$ in pentanoic acid), $1.94\left(\mathrm{~s}, 3 \mathrm{H}, \mathrm{CH}_{3}-\mathrm{Thy}\right), 1.70(\mathrm{~s}, 3 \mathrm{H}, \mathrm{H}-30)$, 0.99, 0.98, 0.88, 0.87, 0.78 (all s, 3H each, H-23-H-27). ${ }^{13} \mathrm{C}$ NMR (125 MHz, DMSO): $\delta_{\mathrm{c}} 182.8$ (COOH), 172.8 (C28), 164.2 (C=O-Thy), 151.4 (C-20), 150.9 (C=O-Thy), 146.7 (C-triazol), 136.7 (CH-Thy), 122.3 (CH-triazol), 110.1 (C-Thy), 109.7 (C-29), 85.0 (1'-ribose), 84.3 (4'-ribose), 80.8 (C-3), 61.1 (5'-ribose), 59.3 (3'-ribose), 55.8 (C-5, $\mathrm{CH}_{2}$ in pentanoic acid), 54.4 (C-17), 53.3 ( $\mathrm{CH}_{2}$ in piperazine), 52.7 (C-18), 50.8 (C-9), 46.0 (C-19), 45.4 (C-1), 41.9 (C-14), 40.8 (C-8), 39.6 (C-4), 38.0 ( $\mathrm{CH}_{2}$ in piperazine), 37.4 (C-10, 2'-ribose), 36.8 (C-13), 36.4 (C-2), 35.8 (C-7), 34.4 (C-22), 32.2 (C-16), 31.3 (C-21), 29.6 (C-15), 28.7 (C-24, =C- $\underline{\mathrm{CH}}_{2}-$ ), $26.2\left(\mathrm{CH}_{2}\right.$ in pentanoic acid), 25.7 (C-12), $23.4\left(\mathrm{CH}_{2}\right.$ in pentanoic acid), 21.1 (C-11), 19.3 (C-30), 18.7 (C-6), 16.9 (C-23), 16.8 (C-26), 16.1 (C-25), 14.5 (C27), 12.7 ( $\mathrm{CH}_{3}-$ Thy). Anal. Calcd for $\mathrm{C}_{52} \mathrm{H}_{79} \mathrm{~N}_{7} \mathrm{O}_{8}: \mathrm{C}, 67.14 ; \mathrm{H}, 8.56$. Found: $\mathrm{C}, 67.21, \mathrm{H}, 8.53 \%$. MS: $\mathrm{m} / z$ [M+K] , found $968.48\left[\mathrm{C}_{52} \mathrm{H}_{79} \mathrm{~N}_{7} \mathrm{O}_{8}\right]^{+}$requires 968.56.

[1-(3'-Deoxythymidine)-1H-1,2,3-triazol-4-yl]-\{[N-2 $\alpha$-methyl-3ß-O-(3',3'-dimethylsuccinyl)-lup-20,29-en-28oyl]-N'-(tert-butoxycarbonyl)\}-2-ethylamine (26). White powder $(\mathrm{B}, 0.75 \mathrm{~g}, 73 \%), \mathrm{mp} 278-280{ }^{\circ} \mathrm{C} .[\alpha]_{\mathrm{D}}^{23}-12.9^{\circ}$ (c $\left.1.08 \mathrm{C}_{2} \mathrm{H}_{5} \mathrm{OH}\right)$. IR (solution in $\mathrm{C}_{2} \mathrm{H}_{5} \mathrm{OH}, \mathrm{cm}^{-1}$ ): $3434(\mathrm{NH}), 1697(\mathrm{C}=0) .{ }^{1} \mathrm{H} \mathrm{NMR}(500 \mathrm{MHz}, \mathrm{MeOD}): \delta_{\mathrm{H}} 7.95(\mathrm{~s}$, $1 \mathrm{H}, \mathrm{H}$-Thy), 7.92 (s, 1H, H-triazol), 7.63 (br s, 1H, NH), 6.51 (t, 1H, J $5.0 \mathrm{~Hz}, \mathrm{H}$-1'-ribose), 5.43-5.40 (m, 1H, H-3'ribose), 4.71, 4.59 (both br s, 2H, H-29), 4.51 (d, $1 \mathrm{H}, J 10.0 \mathrm{~Hz}, \mathrm{H}-3), 4.38-4.34$ (m, $1 \mathrm{H}, \mathrm{H}-4^{\prime}$-ribose), 3.93 and 3.78 (1H each, dd, J 10.0, $5.0 \mathrm{~Hz}, \mathrm{H}-5^{\prime}$-ribose), 3.30-3.16 (m, 4H, $\left.-\mathrm{CH}_{2} \mathrm{NH}-,-\mathrm{CH}_{2} \mathrm{NH}-\mathrm{Boc}\right), 3.10-3.07(\mathrm{~m}, 1 \mathrm{H}, \mathrm{H}-$ 19), 2.95-2.52 (m, 4H, CH $2, \mathrm{H}-2^{\prime}$-ribose, =C- $\left.\mathrm{CH}_{2}-\right)$, 2.67 and $2.59\left(1 \mathrm{H}\right.$, each, d, J $\left.15.5 \mathrm{~Hz},-\mathrm{CH}_{2}-\mathrm{CO}-\right), 2.37-0.85$ $\left(\mathrm{m}, 23 \mathrm{H}, \mathrm{CH}, \mathrm{CH}_{2}\right.$ in pentacyclic skeleton and $\left.1 \mathrm{H}, \mathrm{NH}\right), 1.93\left(\mathrm{~s}, 3 \mathrm{H}, \mathrm{CH}_{3}-\mathrm{Thy}\right), 1.70(\mathrm{~s}, 3 \mathrm{H}, \mathrm{H}-30), 1.44\left(\mathrm{~s}, 9 \mathrm{H}, \mathrm{CH}_{3}\right.$ in Boc), 1.27 (br s, 6H, $\left.-\mathrm{C}\left(\mathrm{CH}_{3}\right)_{2}-\right)$, 1.01, 0.96, 0.89, 0.85, 0.84 (all s, 3H each, H-23-H-27). ${ }^{13} \mathrm{C} \mathrm{NMR} \mathrm{(125} \mathrm{MHz,}$ MeOD): $\delta_{c} 182.8$ (COOH), 179.8 (C-28), 174.5 (- $\mathrm{CH}_{2}$ - CO-), 166.5 (C=O-Thy), 158.9 (CONH-Boc), 152.5 (C-20, C=O-Thy), 147.7 (C-triazol), 138.4 (CH-Thy), 124.1 (CH-triazol ), 111.9 (C-Thy), 110.1 (C-29), 86.8 (C-1'-ribose), 
86.6 (4'-ribose), 85.2 (C-3), 80.3 (C in Boc), 62.3 (5'-ribose), 61.0 (3'-ribose), 57.1 (C-17), 57.0 (C-5), 52.1 (C-9), 51.5 (C-18), 48.6 (C-19), 46.5 (C-1), 45.7 (- $\left.\mathrm{CH}_{2}-\mathrm{CO}-\right), 43.7$ (C-14), 42.2 (C-8), 41.5 (C-4), 40.5 (CH (CH $_{2}$ ethylamine), $40.2\left(\mathrm{CH}_{2}\right.$ in ethylamine, $\left.-\mathrm{C}\left(\mathrm{CH}_{3}\right)_{2}-\right)$, 39.5 (2'-ribose), 39.2 (C-13), 39.0 (C-22), 38.5 (C-10), 35.6 (C2, C-7), 34.2 (C-16), 32.2 (C-21), 30.7 (C-15), 29.9 (=C- $\left.-\mathrm{CH}_{2}-\right), 29.1$ (C-24), $29.0\left(-\mathrm{C}\left(\mathrm{CH}_{3}\right)_{2}-\right), 28.9$ ( $\mathrm{CH}_{3}$ in Boc), 27.0 (C-12, $\left.-\mathrm{C}\left(\mathrm{CH}_{3}\right)_{2}-\right)$, 22.3 (C-11), 19.8 (C-30), 19.6 (C-6), 17.9 (C-26), 17.6 (C-23), 17.0 (C-25), 15.3 (C-27), 12.7 ( $\mathrm{CH}_{3}$-Thy). Anal. Calcd for $\mathrm{C}_{56} \mathrm{H}_{85} \mathrm{~N}_{7} \mathrm{O}_{11}: \mathrm{C}, 65.15 ; \mathrm{H}, 8.30$. Found: $\mathrm{C}, 65.21, \mathrm{H}, 8.27 \%$. MS: $\mathrm{m} / z$ [M+Na] $]^{+}$, found $1054.58\left[\mathrm{C}_{56} \mathrm{H}_{85} \mathrm{~N}_{7} \mathrm{O}_{11}\right]^{+}$requires 1054.62.

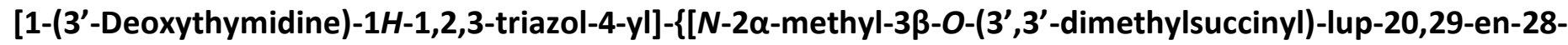
oyl]-N'-(tert-butoxycarbonyl)\}-8-octylamine (27). White powder (B, $1.07 \mathrm{~g}, 96 \%), \mathrm{mp} 192-194{ }^{\circ} \mathrm{C} .[\alpha]_{\mathrm{D}}^{21}-8.45^{\circ}$ (c 0.36, $\mathrm{CH}_{3} \mathrm{OH}$ ). IR (solution in $\mathrm{CH}_{3} \mathrm{OH}, \mathrm{cm}^{-1}$ ): 3433 (NH), 1696 (C=O). ${ }^{1} \mathrm{H} \mathrm{NMR} \mathrm{(500} \mathrm{MHz,} \mathrm{MeOD):} \delta_{\mathrm{H}} 7.94(\mathrm{~s}, 1 \mathrm{H}$, H-Thy), 7.91 (s, 1H, H-triazol), 7.57-7.56 (m, 1H, NH), 6.49 (t, 1H, J 5.0 Hz, H-1'-ribose), 5.43-5.40 (m, 1H, H-3'ribose), 4.71 (br s, 1H, H-29), 4.60-4.59 (m, 2H, H-29, NH), 4.51 (d, 1H, J $10.0 \mathrm{~Hz}, \mathrm{H}-3), 4.37-4.35$ (m, 1H, H-4'ribose), 3.80 and 3.66 (1H each, dd, J 10.0, $5.0 \mathrm{~Hz}, \mathrm{H}-5^{\prime}$-ribose), 3.26-2.58 (m, 9H, $-\mathrm{CH}_{2} \mathrm{NH}-,-\mathrm{CH}_{2} \mathrm{NH}-\mathrm{Boc}, \mathrm{H}-$ 19, $\mathrm{H}-2^{\prime}$-ribose, =C- $\left.\mathrm{CH}_{2}-\right), 2.61$ and $2.58\left(1 \mathrm{H}\right.$, each, d, J $\left.15.5 \mathrm{~Hz},-\mathrm{CH}_{2}-\mathrm{CO}-\right), 2.15-0.73\left(\mathrm{~m}, 23 \mathrm{H}, \mathrm{CH}, \mathrm{CH}_{2}\right.$ in pentacyclic skeleton and $4 \mathrm{H}$ in octylamine), $1.93\left(\mathrm{~s}, 3 \mathrm{H}, \mathrm{CH}_{3}-\mathrm{Thy}\right), 1.70(\mathrm{~s}, 3 \mathrm{H}, \mathrm{H}-30), 1.45$ (br s, 9H, $\mathrm{CH}_{3}$ in $\left.\mathrm{Boc}\right)$, 1.34-1.31 (m, 8H, $\mathrm{CH}_{2}$ in octylamine and $\left.6 \mathrm{H},-\mathrm{C}\left(\mathrm{CH}_{3}\right)_{2}-\right), 1.02,0.96,0.90,0.86,0.84$ (all s, 3H each, $\left.\mathrm{H}-23-\mathrm{H}-27\right)$. ${ }^{13} \mathrm{C}$ NMR (125 MHz, MeOD): $\delta_{\mathrm{C}} 185.2$ (COOH), 179.0 (C-28), 174.0 (- $\mathrm{CH}_{2}-\mathrm{CO}-$ ), 165.0 (C=O-Thy), 158.6 (CONHBoc), 152.5 (C-20), 150.9 (C=O-Thy), 147.5 (C-triazol), 138.4 (CH-Thy), 124.1 (CH-triazol ), 111.9 (C-Thy), 110.1 (C-29), 86.8 (1'-ribose), 86.5 (4'-ribose), 85.2 (C-3), 79.9 (C in Boc), 62.3 (5'-ribose), 61.0 (3'-ribose), 57.1 (C17), 56.9 (C-5), 52.1 (C-9), 51.5 (C-18), 48.2 (C-19), 46.3 (C-1), 45.7 (- $\mathrm{CH}_{2}-\mathrm{CO}-$ ), 43.7 (C-14), 42.2 (C-8), 41.5 (C4), 40.5 ( $\mathrm{CH}_{2}$ in octylamine), $40.2\left(-\mathrm{C}\left(\mathrm{CH}_{3}\right)_{2}-, \mathrm{CH}_{2}\right.$ in octylamine), 39.6 (2'-ribose), 39.1 (C-13), 38.9 (C-22), 38.5 (C-10), 35.6 (C-7), 35.5 (C-2), 34.3 (C-16), 32.1 (C-21), 30.8 (C-15), 30.6 ( $\mathrm{CH}_{2}$ in octylamine), $30.1\left(\mathrm{CH}_{2}\right.$ in octylamine), 29.9 (=C- $\left.\underline{\mathrm{CH}}_{2}-\right), 29.1$ (C-24), $29.0\left(-\mathrm{C}\left(\underline{\mathrm{CH}}_{3}\right)_{2}-\right), 28.9\left(\mathrm{CH}_{3}\right.$ in Boc), $28.2\left(\mathrm{CH}_{2}\right.$ in octylamine), $28.0\left(\mathrm{CH}_{2}\right.$ in octylamine), 27.1 (C-12), $27.0\left(-\mathrm{C}\left(\underline{\mathrm{CH}}_{3}\right)_{2}-\right), 22.3$ (C-11), 19.9 (C-30), 19.6 (C-6), 17.9 (C-26), 17.7 (C-23), 17.0 (C-25), 15.3 (C-27), 12.69 ( $\mathrm{CH}_{3}$-Thy). Anal. Calcd for $\mathrm{C}_{62} \mathrm{H}_{97} \mathrm{~N}_{7} \mathrm{O}_{11}$ : C, 66.70; $\mathrm{H}, 8.76$. Found: $\mathrm{C}, 66.64, \mathrm{H}, 8.69 \%$. MS: $\mathrm{m} / \mathrm{z}[\mathrm{M}+\mathrm{K}]^{+}$, found $1154.50\left[\mathrm{C}_{62} \mathrm{H}_{97} \mathrm{~N}_{7} \mathrm{O}_{11}\right]^{+}$requires 1154.69 .

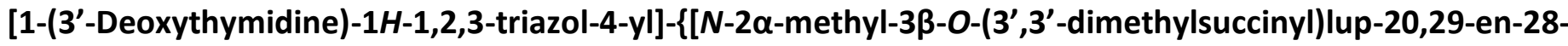

oyl)-2-aminoethyl]carbamoyl\}methane (28). White powder $(\mathrm{B}, 0.58 \mathrm{~g}, 60 \%), \mathrm{mp} 248-250{ }^{\circ} \mathrm{C} .[\alpha]_{\mathrm{D}}^{22}-2.2^{\circ}(c$ 0.36, $\mathrm{C}_{2} \mathrm{H}_{5} \mathrm{OH}$ ). IR (solution in $\left.\mathrm{C}_{2} \mathrm{H}_{5} \mathrm{OH}, \mathrm{cm}^{-1}\right): 1714(\mathrm{C}=\mathrm{O}) .{ }^{1} \mathrm{H}$ NMR $(500 \mathrm{MHz}, \mathrm{MeOD}): \delta_{\mathrm{H}} 7.96(\mathrm{~s}, 1 \mathrm{H}, \mathrm{H}-\mathrm{Thy})$, 7.92 (s, 1H, H-triazol), 7.65 (m, 1H, NH), 6.50 (t, 1H, J $5.0 \mathrm{~Hz}, \mathrm{H}$-1'-ribose), 5.45-5.43 (m, 1H, H-3'-ribose), 4.70, 4.59 (both br s, 2H, H-29), 4.50 (d, $1 \mathrm{H}, J 10.0 \mathrm{~Hz}, \mathrm{H}-3), 4.36-4.34\left(\mathrm{~m}, 1 \mathrm{H}, \mathrm{H}-4^{\prime}\right.$-ribose), 3.93 and 3.80 (1H each, dd, J 10.0, $5.0 \mathrm{~Hz}, \mathrm{H}-5^{\prime}$-ribose), 3.33-3.29 (m, 4H, $\left.-\mathrm{CH}_{2} \mathrm{NH}-, \mathrm{CH}_{2} \mathrm{NHCOCH}_{3}\right), 3.10-3.06$ (m, 1H, H-19), $2.96-2.94$ (m, $1 \mathrm{H}, \mathrm{H}-2^{\prime}$-ribose), 2.84-2.53 (m, 3H, =C- $\mathrm{CH}_{2-}, \mathrm{H}-2^{\prime}$-ribose), 2.67 and 2.58 (1H each, d, J 15.5 Hz, $-\mathrm{CH}_{2}-\mathrm{CO}-$ ), 2.39-0.72 (m, 23H, CH, CH in pentacyclic skeleton and $1 \mathrm{H}, \mathrm{NH}), 1.96\left(\mathrm{~s}, 3 \mathrm{H},-\mathrm{NHCOCH}_{3}\right), 1.92\left(\mathrm{~s}, 3 \mathrm{H}, \mathrm{CH}_{3}-\mathrm{Thy}_{2}\right.$ ), 1.69 (s, 3H, H-30), 1.27 (br s, 6H, $-\mathrm{C}\left(\mathrm{CH}_{3}\right)_{2}-$ ), 1.01, 0.94, 0.89, 0.85, 0.83 (all s, 3H each, H-23-H-27). ${ }^{13} \mathrm{C} \mathrm{NMR}$ (125 MHz, MeOD): $\delta_{C} 185.0$ (COOH), 179.6 (C-28), $174.3\left(-\mathrm{CH}_{2}-\underline{\mathrm{CO}}-\right), 173.8\left(\mathrm{NHCOCH}_{3}\right), 166.5$ (C=O-Thy), 152.5 (C-20), 152.4 (C=O-Thy), 147.4 (C-triazol), 138.4 (CH-Thy), 124.1 (CH-triazole), 111.8 (C-Thy), 110.2 (C-29), 86.8 (1'-ribose), 86.6 (4'-ribose), 85.1 (C-3), 62.3 (5'-ribose), 60.9 (3'-ribose), 57.1 (C-17), 57.0 (C-5), 52.0 (C-9), 51.5 (C-18), 48.6 (C-19), 46.4 (C-1), 45.8 (- $\left.\mathrm{CH}_{2}-\mathrm{CO}-\right), 43.7$ (C-14), 42.2 (C-8), 40.5 ( $\mathrm{CH}_{2}$ in ethylamine), 40.5 (C-4), $40.2\left(\mathrm{CH}_{2}\right.$ in ethylamine, $\left.-\underline{\mathrm{C}}\left(\mathrm{CH}_{3}\right)_{2}-\right)$, 39.5 (2'-ribose), 39.2 (C-13), 39.0 (C-22), 38.5 (C-10), 35.5 (C-2, C-7), 34.2 (C-16), 32.0 (C-21), 30.7 (C-15), 29.9 (=C- $\left.\underline{\mathrm{CH}}_{2}-\right), 29.1$ (C-24), 27.0 (C-12), $26.5\left(-\mathrm{C}\left(\underline{\mathrm{CH}}_{3}\right)_{2}-\right), 22.9(\mathrm{NHCOCH} 3), 22.3$ (C-11), 19.9 (C-30), 19.6 (C-6), 17.9 (C-23), 17.7 (C-26), 16.9 (C-25), 15.3 (C-27), 12.7 ( $\mathrm{CH}_{3}$-Thy). Anal. Calcd for $\mathrm{C}_{53} \mathrm{H}_{79} \mathrm{~N}_{7} \mathrm{O}_{10}: \mathrm{C}, 65.34 ; \mathrm{H}, 8.17$. Found: $\mathrm{C}, 65.29, \mathrm{H}, 8.23 \%$. MS: $m / z[\mathrm{M}+\mathrm{Na}]^{+}$, found $995.95\left[\mathrm{C}_{53} \mathrm{H}_{79} \mathrm{~N}_{7} \mathrm{O}_{10}\right]^{+}$ requires 996.58 . 


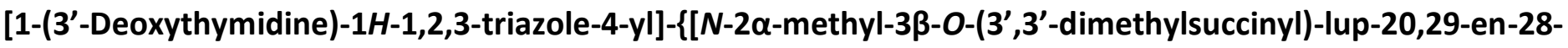
oyl)-8-aminooctyl]carbamoyl\}methane (29). White powder (B, $0.68 \mathrm{~g}, 64 \%), \mathrm{mp} 194-196{ }^{\circ} \mathrm{C} .[\alpha]_{\mathrm{D}}^{21}-8.3^{\circ}(\mathrm{c}$ 0.36, $\mathrm{CH}_{3} \mathrm{OH}$ ). IR (solution in $\mathrm{CH}_{3} \mathrm{OH}, \mathrm{cm}^{-1}$ ): 1732 (C=O). ${ }^{1} \mathrm{H}$ NMR (500 MHz, MeOD ): $\delta_{\mathrm{H}} 7.92$ (s, $\left.1 \mathrm{H}, \mathrm{H}-\mathrm{Thy}\right), 7.87$ (s, 1H, H-triazol), 5.56 (m, 1H, NH), 6.46 (t, 1H, J 5.0 Hz, H-1'-ribose), 5.40-5.42 (m, 1H, H-3'-ribose), 4.69, 4.56 (both br s, 2H, H-29), 4.48 (d, 1H, J $10.0 \mathrm{~Hz}, \mathrm{H}-3), 4.35-4.33\left(\mathrm{~m}, 1 \mathrm{H}, \mathrm{H}-4^{\prime}\right.$-ribose), 3.90 and 3.77 (1H each, dd, J 10.0, $5.0 \mathrm{~Hz}, \mathrm{H}-5^{\prime}$-ribose), 3.26-3.23 (m, $\left.1 \mathrm{H}, \mathrm{H}-19\right), 3.15-3.03\left(\mathrm{~m}, 4 \mathrm{H},-\mathrm{CH}_{2} \mathrm{NH}-, \mathrm{CH}_{2} \mathrm{NHCOCH}_{3}\right), 2.93-2.56(\mathrm{~m}$, $4 \mathrm{H},=\mathrm{C}-\mathrm{CH}_{2}-, \mathrm{H}-2^{\prime}$-ribose), 2.65 and 2.58 (1H each, d, J $\left.15.0 \mathrm{~Hz},-\mathrm{CH}_{2}-\mathrm{CO}-\right)$, 2.38-0.69 (m, 23H, CH, $\mathrm{CH}_{2}$ in pentacyclic skeleton and $1 \mathrm{H}, \mathrm{NH}), 1.90\left(\mathrm{~s}, 3 \mathrm{H},-\mathrm{NHCOCH}_{3}\right), 1.91\left(\mathrm{~s}, 3 \mathrm{H}, \mathrm{CH}_{3}-\mathrm{Thy}\right), 1.67(\mathrm{~s}, 3 \mathrm{H}, \mathrm{H}-30), 1.33-1.31$ (m, $12 \mathrm{H}, \mathrm{CH}_{2}$ in octylamine), 1.26 (br s, $\left.6 \mathrm{H},-\mathrm{C}\left(\mathrm{CH}_{3}\right)_{2}-\right), 0.99,0.93,0.87,0.83,0.81$ (all s, 3H each, $\mathrm{H}-23-\mathrm{H}-27$ ). ${ }^{13} \mathrm{C}$ NMR (125 MHz, MeOD): $\delta \mathrm{c} 183.6$ (C-28), 183.6 (COOH), $174.0\left(-\mathrm{CH}_{2}-\underline{\mathrm{CO}}-\right), 173.2\left(\mathrm{NHCOCH}_{3}\right), 166.5$ (C=OThy), 152.5 (C-20, C=O-Thy), 147.4 (C-triazol), 138.4 (CH-Thy), 124.1 (CH-triazol ), 111.8 (C-Thy), 110.1 (C-29), 86.8 (1'-ribose), 86.6 (4'-ribose), 85.1 (C-3), 62.3 (5'-ribose), 61.0 (3'-ribose), 57.0 (C-5, C-17), 52.1 (C-9), 51.6 (C-18), 48.2 (C-19), 46.3 (C-1), 45.6 (- $\left.\mathrm{CH}_{2}-\mathrm{CO}-\right), 43.7$ (C-14), 42.2 (C-8), 40.7 (C-4), 40.7 ( $\mathrm{CH}_{2}$ in octylamine), $40.3\left(-\underline{C}\left(\mathrm{CH}_{3}\right)_{2}-\right)$, 40.2 (2'-ribose), 39.6 (C-13), 39.1 ( $\mathrm{CH}_{2}$ in octylamine), 39.0 (C-22), 38.5 (C-10), 35.7 (C-2), 35.6 (C-7), 34.4 (C-16), 32.1 (C-21), 30.7 (C-15, $\mathrm{CH}_{2}$ in octylamine), 30.5 ( $\mathrm{CH}_{2}$ in octylamine), $30.4\left(\mathrm{CH}_{2}\right.$ in octylamine), 29.8 (=C- $\left.\mathrm{CH}_{2}-\right), 29.1$ (C-24), $28.2\left(\mathrm{CH}_{2}\right.$ in octylamine), 28.1 ( $\mathrm{CH}_{2}$ in octylamine), 27.1 (C-12), 26.8 ($\left.\mathrm{C}\left(\underline{\mathrm{CH}}_{3}\right)_{2}-\right), 26.4\left(-\mathrm{C}\left(\underline{\mathrm{CH}}_{3}\right)_{2}-\right), 22.7\left(\mathrm{NHCOCH}_{3}\right), 22.3$ (C-11), 19.8 (C-30), 19.6 (C-6), 17.9 (C-26), 17.6 (C-23), 17.0 (C-25), 15.3 (C-27), 12.7 ( $\mathrm{CH}_{3}$-Thy). Anal. Calcd for $\mathrm{C}_{59} \mathrm{H}_{91} \mathrm{~N}_{7} \mathrm{O}_{10}$ : C, 66.95; H, 8.67. Found: $\mathrm{C}, 66.88, \mathrm{H}, 8.71 \%$. $\mathrm{MS}: \mathrm{m} / z[\mathrm{M}+\mathrm{K}]^{+}$, found $1096.53\left[\mathrm{C}_{59} \mathrm{H}_{91} \mathrm{~N}_{7} \mathrm{O}_{10}\right]^{+}$requires 1096.65.

\section{Acknowledgements}

The authors are deeply thankful to the staff members of the analytical center "Agidel" at the Institute of Petrochemistry and Catalysis of RAS for spectral measurement. This work was performed with financial support from the Russian Science Foundation (Grant №. 16-13-10051).

\section{Supplementary Material}

Scans of the ${ }^{1} \mathrm{H}$ NMR and ${ }^{13} \mathrm{C}$ NMR spectra of all new compounds.

\section{References}

1. Cichewicz, R. H.; Kouzi, S. A. Med. Res. Rev. 2004, 24, 90.

http://dx.doi.org/10.1002/med.10053

2. Alakurtti, S.; Makela, T.; Koskimies, S.; Yli-Kauhaluoma, J. Eur. J. Pharm. Sci. 2006, $29,1$.

http://dx.doi.org/10.1016/i.ejps.2006.04.006

3. Csuk, R. Expert Opin. Ther. Pat. 2014, 24, 913. http://dx.doi.org/10.1517/13543776.2014.927441

4. Yogeeswari, P.; Sriram, D. Curr. Med. Chem. 2005, 12, 657. http://dx.doi.org/10.2174/0929867053202214 
5. Kashiwada, Y.; Hashimoto, F.; Cosentino, L. M.; Chen, C.-H.; Garrett, P. E.; Lee, K.-H. J. Med. Chem. 1996, 39, 1016.

http://dx.doi.org/10.1021/jm950922q

6. Hashimoto, F.; Kashiwada, Y.; Cosentino, L. M.; Chen, C.-H.; Garrett, P. E.; Lee, K.-H. Bioorg. Med. Chem. 1997, 5, 2133.

http://dx.doi.org/10.1016/S0968-0896(97)00158-2

7. Aiken, C.; Chen, C. Trends Mol. Med. 2005, 11, 31.

http://dx.doi.org/10.1016/j.molmed.2004.11.001

8. Martin, D. E.; Salzwedel, K.; Allaway, G. P. Antivir. Chem. Chemother. 2008, 19, 107. http://dx.doi.org/10.1177/095632020801900301

9. Kashiwada, Y.; Sekiya, M.; Ikeshiro, Y.; Fujioka, T.; Kilgore, N. R.; Wild, C. T.; Allaway, G. P.; Lee, K. H. Bioorg \& Med. Chem. Lett. 2004, 14, 5851.

http://dx.doi.org/10.1016/j.bmcl.2004.09.033

10. Yu, D.; Morris-Natschke, S. L.; Lee, K. H. Med. Res. Rev. 2007, 27, 108. http://dx.doi.org/10.1002/med.20075

11. Yu, D.; Wild, C. T.; Martin, D. E.; Morris-Natschke, S. L.; Chen, C. H.; Allaway, G. P.; Lee, K. H. Expert Opin. Investig. Drugs, 2005, 14, 681.

http://dx.doi.org/10.1517/13543784.14.6.681

12. Qian, K.; Bori, I. D.; Chen, C.-H.; Huang, L.; Lee, K.-H. J. Med. Chem. 2012, 55, 8128. http://dx.doi.org/10.1021/im301040s

13. Holz-Smith, S. L.; Sun, I. C.; Jin, L.; Matthews, T. J.; Lee, K. H.; Chen, C. H. Antimicrob Agents Chemother, 2001, 45, 60 .

http://dx.doi.org/10.1128/AAC.45.1.60-66.2001

14. Sun, I.-C.; Chen, C.-H.; Kashiwada, Y.; Wu, J.-H.; Wang, H.-K.; Lee, K.-H. J. Med. Chem. 2002, 45, 4271. http://dx.doi.org/10.1021/jm020069c

15. Huang, L.; Yuan, X.; Aiken, C.; Chen, C. H. Antimicrob. Agents Chemother. 2004, 48, 663. http://dx.doi.org/10.1128/AAC.48.2.663-665.2004

16. Huang, L.; Ho, P.; Lee, K.-H.; Chen, C.-H. Bioorg. Med. Chem. 2006, 14, 2279. http://dx.doi.org/10.1016/j.bmc.2005.11.016

17. Qian, K.; Yu, D.; Chen, C.-H.; Huang, L.; Morris-Natschke, S. L.; Nitz, T. J.; Salzwedel, K.; Reddick, M.; Allaway, G. P.; Lee, K.-H. J. Med. Chem. 2009, 52, 3248.

http://dx.doi.org/10.1021/jm900136j

18. Dang, Z.; Ho, P.; Zhu, L.; Qian, K.; Lee, K.-H.; Huang, L.; Chen, C.-H. J. Med. Chem. 2013, 56, 2029. http://dx.doi.org/10.1021/jm3016969

19. Zhao, Y.; Gu, Q.; Morris-Natschke, S. L.; Chen, C.-H.; Lee, K.-H. J. Med. Chem. 2016, 59, 9262. http://dx.doi.org/10.1021/acs.jmedchem.6b00461

20. Xiong, J.; Kashiwada, Y.; Chen, C.-H.; Qian, K.; Morris-Natschke, S. L.; Lee, K.-H.; Takaishi, Y. Bioorg. Med. Chem. 2010, 18, 6451.

http://dx.doi.org/10.1016/i.bmc.2010.06.092

21. Bori, I. D.; Hung, H. Y.; Qian, K.; Chen, C. H.; Morris-Natschke, S. L.; Lee, K. H. Tetrahedron Lett. 2012, 53, 1987.

22. http://dx.doi.org/10.1016/i.tetlet.2012.02.022

23. Dang Thi, T. A.; Kim Tuyet, N. T.; Pham The, C.; Thanh Nguyen, H.; Ba Thi, C.; Doan Duy, T.; D’hooghe, M.; Van Nguyen, T. Bioorg. Med. Chem. Lett. 2014, 24, 5190.

http://dx.doi.org/10.1016/j.bmcl.2014.09.079 
24. Dang Thi, T. A.; Kim Tuyet, N. T.; Pham The, C.; Thanh Nguyen, H.; Ba Thi, C.; Thi Phuong, H.; Van Boi, L.; Van Nguyen, T.; D’hooghe, M. Tetrahedron Lett. 2015, 56, 218. http://dx.doi.org/10.1016/j.tetlet.2014.11.069

25. Spivak, A. Y.; Gubaidullin, R. R.; Galimshina, Z. R.; Nedopekina, D. A.; Odinokov, V. N. Tetrahedron, 2016, $72,1249$. http://dx.doi.org/10.1016/j.tet.2016.01.024

26. Spivak, A. Y.; Galimshina, Z. R.; Nedopekina, D. A.; Odinokov, V. N. Chemistry of Natural Compounds, 2018, $54,315$. http://dx.doi.org/10.1007/s10600-018-2331-1

27. Rathi, A. K.; Syed, R.; Shin, H. S.; Patel, R. V. Expert Opin. Ther. Pat., 2016, 26, 777. http://dx.doi.org/10.1080/13543776.2016.1189902

28. Patel, R. V.; Park, S. W. Mini Rev. Med. Chem. 2013, 13, 1579. http://dx.doi.org/10.2174/13895575113139990073

29. Gao, P.; Sun, L.; Zhou, J.; Li, X.; Zhan, P.; Liu X. Expert Opin. Drug Discov. 2016, 11, 857. http://dx.doi.org/10.1080/17460441.2016.1210125

30. Coulibaly, W. K.; Paquin, L.; Bénie, A.; Békro, Y.-A.; Guével R. L.; Ravache, M.; Corlu, A.; Bazureau, J. P. Med. Chem. Res. 2014, 24, 1653. http://dx.doi.org/10.1007/s00044-014-1186-7 